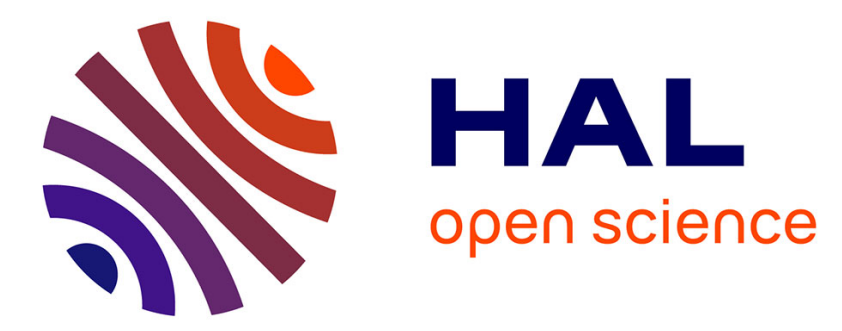

\title{
Audio-visual speech scene analysis: Characterization of the dynamics of unbinding and rebinding the McGurk effect
}

Olha Nahorna, Frédéric Berthommier, Jean-Luc Schwartz

\section{To cite this version:}

Olha Nahorna, Frédéric Berthommier, Jean-Luc Schwartz. Audio-visual speech scene analysis: Characterization of the dynamics of unbinding and rebinding the McGurk effect. Journal of the Acoustical Society of America, 2015, 137 (1), pp.362-377. 10.1121/1.4904536 . hal-01213897

\author{
HAL Id: hal-01213897 \\ https://hal.science/hal-01213897
}

Submitted on 9 Oct 2015

HAL is a multi-disciplinary open access archive for the deposit and dissemination of scientific research documents, whether they are published or not. The documents may come from teaching and research institutions in France or abroad, or from public or private research centers.
L'archive ouverte pluridisciplinaire $\mathbf{H A L}$, est destinée au dépôt et à la diffusion de documents scientifiques de niveau recherche, publiés ou non, émanant des établissements d'enseignement et de recherche français ou étrangers, des laboratoires publics ou privés. 
GIPSA-Lab, Speech and Cognition Department, 


\section{Abstract}

19 While audiovisual interactions in speech perception have long been considered as 20 automatic, recent data suggest that this is not the case. In a previous study, Nahorna et 21 al. (2012) [J. Acoust. Soc. Am, 132, 1061-1077] showed that the McGurk effect is

22 reduced by a previous incoherent audiovisual context. This was interpreted as showing 23 the existence of an audiovisual binding stage controlling the fusion process. Incoherence 24 would produce unbinding and decrease the weight of the visual input in fusion. The present paper explores the audiovisual binding system to characterize its dynamics. A first experiment assesses the dynamics of unbinding, and shows that it is rapid: an

27 incoherent context less than 0.5 s long (typically one syllable) suffices to produce a 28 maximal reduction in the McGurk effect. A second experiment tests the rebinding process, by presenting a short period of either coherent material or silence after the incoherent unbinding context. Coherence provides rebinding, with a recovery of the

31 McGurk effect, while silence provides no rebinding and hence freezes the unbinding 32 process. These experiments are interpreted in the framework of an audiovisual speech

33 scene analysis process assessing the perceptual organization of an audiovisual speech input before decision takes place at a higher processing stage.

Main section: 43.71 
39 Keywords: audiovisual speech perception; multisensory coherence; conditional binding;

40 attentional mechanisms; audiovisual fusion 


\section{I. Introduction}

\section{A. The standard model of audiovisual fusion in speech perception}

44 Audiovisual interactions in speech perception are generally described as an unconditional

45 fusion process in the sense that (1) visual and auditory modalities would be translated

46 into a common format and/or converge towards a given representational stage, where

47 the entries would be merged in a way still to define, and (2) this merging process would

48 be automatic, depending neither on the input stimuli nor on the context and in particular 49 not on possible attentional effects. In other words, if $I_{A}$ and $I_{V}$ are respectively the

50 auditory and visual inputs at time $t$, audiovisual perception would be described by the 51 following process:

$52 \quad \mathrm{P}_{\mathrm{AV}}(\mathrm{t})=\mathrm{F}\left(\mathrm{I}_{\mathrm{A}}, \mathrm{I}_{\mathrm{V}}\right)$

53 where $P_{A V}(t)$ is the percept at time $t$, and $F$ is a fusion function whose output exclusively

54 depends on inputs $\mathrm{I}_{\mathrm{A}}$ and $\mathrm{I}_{\mathrm{V}}$.

This framework provided the basis for explaining the results of the two main paradigms

for the study of audiovisual interactions: speech perception in noisy conditions, in which the visual input enhances the intelligibility of auditory input degraded by acoustic noise (Sumby and Pollack, 1954; Erber, 1969; Benoît et al. 1994); and the McGurk effect, in which two conflicting inputs (typically an audio "b" and a video "g") are combined into a specific fused percept, typically "th" or "d" (McGurk and MacDonald, 1976) .

61 The literature in the 80s and 90s was mainly focused on specifying the nature of the $\mathrm{F}$ operator in (Eq. 1), and in particular on the two components of this operator: (1) the nature of the common representation towards which the auditory and visual inputs would converge before fusion, and (2) the mathematical content of the fusion operator. 
The first question involved assumptions about auditory vs. motor recoding and the issue about early fusion (combination of sensory inputs recoded into a common prephonological format before decision occurs) vs. late fusion (separate classification of sensory inputs followed by a decision fusion process, operating in a common space of phonetic or phonological features): see reviews in Summerfield (1987) and Schwartz et al. (1998). Concerning the second question, Massaro's group extensively studied the fusion operator content. They proposed the Fuzzy-Logical Model of Perception (FLMP) and presented systematic comparison of possible operators competing with the optimal fusion operator realized by a multiplicative process in the FLMP (Massaro and Cohen, 1983; Massaro, 1987, 1989).

\section{B. Non-automaticity of the fusion process}

While the fusion process has long been considered as automatic (Massaro, 1987; SotoFaraco et al., 2004), works in the 90s and 2000s displayed various departures from this hypothesis in several directions.

This began with the issue whether the fusion process might depend on the subject and especially her/his culture and language. The pioneer experiments by Sekiyama and Tohkura (1991, 1993) displayed lesser McGurk effect in Japanese compared to American English and generated many studies and much debate in the 90s (e.g. Massaro et al., 1993; Furster-Duran, 1996). It has however been obscured by methodological problems associated with model comparison in an audiovisual perception experiment, since it is difficult to disentangle what comes from unisensory perception (i.e. how subjects perceive each input independently of the other) and what is actually due to fusion. We recently showed how the use of a rigorous methodological framework for comparing models 
89 (Schwartz, 2006) enables to confirm the existence of differences between subjects, some Later, experiments by Soto-Faraco' group showed that an attentional load applied to the

where S represents the subject with her/his own specificities, both individual ("auditory" vs. "visual subjects") and possibly cultural or linguistic (Sekiyama and Burnham, 2008).

The second direction was provided in the 2000s by experiments showing the potential role of attentional effects. In the "face-leaf" study by Tiippana et al. (2004), a visual distractor (a transparent leaf gently moving on the speaking face) superimposed on a conflicting audiovisual stimulus (such as seeing the face of a female speaker uttering " $\mathrm{k}$ ", superimposed on a "p" sound) decreased the McGurk effect (with fewer fusion responses " $t$ " and more auditory responses "p"). The authors' interpretation was that the participants attributed less weight to the visual modality in the fusion process because the leaf distracted their visual attention (see also Andersen et al., 2001). Once again, the use of a rigorous mathematical framework enabled to confirm this interpretation (Schwartz et al, 2010) by introducing an attentional factor in the fusion process. This could be formalized by the following equation:

$\mathrm{P}_{\mathrm{AV}}(\mathrm{t})=\mathrm{F}\left(\mathrm{I}_{\mathrm{A}}, \mathrm{I}_{\mathrm{V}}, \mathrm{S}, \mathrm{A}\right)$

where A represents a global attentional factor, modulated in the leaf-face experiment by the visual distractor reducing the weight of the $I_{V}$ visual input in the fusion process.

\section{1 fusion process (consisting in superposing to the McGurk audiovisual speech perception}


112 task an additional task involving the processing of other auditory, visual or tactile stimuli:

113 Alsius et al., 2005, 2007) decreased the McGurk effect. The authors concluded that the

114 fusion process was not automatic, but rather under the control of a global attentional 115 process modulated by the attentional load. In the framework of (Eq. 3), it could be 116 suggested that the attentional load factor is integrated inside the A term, resulting in a 117 decrease of the weight of the $\mathrm{I}_{\mathrm{V}}$ visual input in the fusion process.

118 The passage from (Eq. 1) to (Eq. 3) can be computationally implemented in various 119 ways. We ourselves proposed an implementation based on the late-fusion multiplicative 120 FLMP model where fusion only depends on the unisensory inputs, in accordance with 121 Eq. 1. From that basis, we introduced a weighted fuzzy-logical model of perception, 122 WFLMP, in which fusion would also involve specific weights controlling the role of each 123 modality in the fusion process. This led to various implementation of the WFLMP, in 124 which weights depend on the subject's individual characteristics (Schwartz, 2010; Huyse 125 et al., 2013), attentional processes (Schwartz et al., 2010), or degradation of the auditory 126 or visual input (Heckmann et al., 2002; Huyse et al., 2013).

\section{Audio-Visual Speech Scene Analysis and the binding and fusion hypothesis}

129 A remarkable point in the studies by Tiippana et al. (2004) and Alsius et al. (2005) is that 130 the subjects were simultaneously processing multiple auditory or visual inputs (see also 131 Andersen et al., 2009; Alsius and Soto-Faraco, 2011). Then a question arises: how do 132 subjects succeed in segregating mixed sources in each unisensory flow before attempting 133 to fuse the adequate pieces of information? This is the issue of perceptual scene analysis. 134 The concept of auditory scene analysis (ASA) popularized by Bregman (1990) has largely renewed our understanding of auditory processing, gradually imposing a model in which 
136 a perceptual organization stage should intervene in the auditory categorization process by specifying the different sources of information mixed in the scene before they could be efficiently identified. Auditory scene analysis involves segmenting the scene into sensory elements that should be grouped in respect to their common source, either by bottom-up

140 innate primitives or by learnt top-down schemas. The way various primitives, likely 141 detected in different auditory maps in the human brain, are grouped together to form a 142 whole percept is generally called the binding problem.

143 A multisensory scene such as a mixture of audiovisual speech sources contains both 144 acoustic and optic cues, likely resulting in auditory and visual primitives. The question 145 addressed by our group since a number of years concerns whether audiovisual scenes, 146 including multiple audiovisual speech streams, could involve an Audio-Visual Speech 147 Scene Analysis process in which auditory and visual primitives would be adequately 148 bound together before audiovisual fusion could occur. Studies in this area are rare, and 149 the classical conception is rather that monosensory grouping precedes multisensory 150 interactions, with a number of data in support of this view (Sanabria et al., 2005; Keetels 151 et al., 2007). However, some data suggest that audiovisual interactions could intervene at 152 various stages of the speech decoding process.

153 This includes the audiovisual speech detection advantage in which the presence of the 154 speaker's face has been shown to improve the detection of speech embedded in acoustic 155 noise (Grant and Seitz, 2000) and produce specific gains in intelligibility (Schwartz et al., 156 2004). The audiovisual speech detection advantage happens to operate independently of 157 the possibility to understand speech, even in a foreign language (Kim and Davis, 2003) or 158 with time-reverse speech. The temporal correlation between the auditory and visual 159 components plays a crucial role in this process (Kim and Davis 2004). On the other way 160 round, an auditory stimulus comodulated with the visual stimulus of a talking face 
161 improves the visibility of the talking face masked by interocular suppression (Alsius and

162 Munhall, 2013). In all these studies, it is suggested that audiovisual comodulation

163 provides a binding process able to fuse together acoustic and optic cues, improving the

164 detection of an audiovisual source or the extraction of audiovisual cues masked by 165 auditory or visual noise.

166 Furthermore, electrophysiological experiments display early audiovisual interactions in 167 the auditory cortex (Colin et al., 2002; Besle et al., 2004), showing that visual speech can 168 speed up the cortical processing of the auditory input as soon as $100 \mathrm{~ms}$ after the stimulus 169 onset (van Wassenhove et al., 2005). Altogether, these data suggest that the visual speech 170 flow could modulate ongoing auditory feature processing at various levels (Bernstein et 171 al., 2004; Bernstein et al., 2008; Arnal et al., 2009; Eskelund et al., 2011).

172 This led Berthommier (2004) propose a two-stage model in which audiovisual coherence 173 between the auditory and the visual input would be computed prior to fusion, to 174 determine whether the two inputs are coherent and hence should be bound together and 175 produce perceptual fusion. This binding and fusion process would consist in conditioning 176 fusion on binding, just as Bregman reasoned that auditory perception should be 177 conditioned by auditory binding thanks to an auditory scene analysis process. It may be 178 described by an additional expansion of (Eq. 3):

$179 \quad \mathrm{P}_{\mathrm{AV}}(\mathrm{t})=\mathrm{F}\left(\mathrm{I}_{\mathrm{A}}, \mathrm{I}_{\mathrm{V}}, \mathrm{S}, \mathrm{A}, \mathrm{C}_{\mathrm{AV}}\right)$

180 wherein $\mathrm{C}_{\mathrm{AV}}$ represents an audiovisual coherence index enabling the subject estimate 181 whether the auditory and visual inputs should be fused or not.

182 This assumption found an experimental support in a series of experiments that we 183 conducted recently (Nahorna et al., 2012). In these experiments, we manipulated the 
184 audiovisual coherence index $\mathrm{C}_{\mathrm{AV}}$ by providing an audiovisual context prior to the

185 McGurk target. The context was either coherent (auditory and visual inputs from the

186

188

189

190

191 same source, namely a speaker producing a series of audiovisual syllables) or incoherent (auditory and visual input from two different sources, for example the sound of the speaker producing a sequence of acoustic syllables, dubbed on the image of the speaker producing a sequence of sentences unrelated with the sequence of acoustic syllables). There were two targets, one congruent (audiovisual "ba") and one incongruent (the McGurk target made of an auditory "ba" with a visual "ga"). The subject's task consisted in attempting to detect online "ba" or "da" syllables inside a film made of a series of such (context + target) sequences, without knowing when they would occur in the film. The online monitoring procedure aimed at emphasizing the role of audiovisual scene analysis processes, the assumption being that with incoherent context, the subject would unbind to a certain extent the auditory and visual streams and hence display less McGurk effect, with more "ba" and less "da" responses to McGurk targets. It appeared that the McGurk effect was indeed largely reduced in the incoherent context condition in respect with the coherent context condition.

We interpreted these results in the binding and fusion framework, by assuming that:

(1) Without context, the subjects would be in a default state where pieces of information are bound together, as it seems to be the case for auditory scene analysis (see e.g. Bregman \& Pinker 1978), and also for visual scene analysis (Hupé and Pressnitzer, 2011). Therefore the auditory and visual inputs are supposedly coherent and hence bound together;

(2) Subjects would estimate the audiovisual coherence index $\mathrm{C}_{\mathrm{AV}}$ by the context. In the incoherent context condition, this index suggests that sound and image should not be bound together, which would decrease the role of the visual input in the 
fusion process and hence decrease the amount of McGurk responses. This was called by Nahorna et al. (2012) unbinding;

(3) In the coherent context condition on the contrary, the index would confirm that sound and image should be bound together, hence the subject would stay in the default state and display a stable McGurk effect.

D. Dynamics of the binding process in audiovisual speech scene analysis

216 We assume that the computation of the audiovisual coherence $C_{A V}$ index is part of a 217 general scene analysis process, generalizing Bregman' ASA to audiovisual scenes. We 218 therefore consider that a major issue of current research on audiovisual fusion in speech 219 perception is the characterization of this binding and fusion process, and more generally 220 the understanding of what constitutes the audiovisual speech scene analysis system.

221 In this paper we capitalize on the "context + target" experimental paradigm developed by 222 Nahorna et al. (2012) to focus on the dynamics of the binding-unbinding process, around 223 two major questions.

\section{1. Time constant of the unbinding process}

225 The first one deals with the precise time constant of the unbinding process. The 226 experiments in our previous work used rather long contextual stimuli, from around $3 \mathrm{~s}$ to 227 around $10 \mathrm{~s}$. It appeared that the amount of unbinding - displayed by the amount of 228 decrease in the McGurk effect - was constant over this duration range. While McGurk 229 stimuli in a coherent context were identified as "ba" $60 \%$ to $70 \%$ of the time and as "da" 230 the remaining $40 \%$ to $30 \%$, the application of an incoherent context decreased the 231 amount of "da" responses to about the half of their value without context, independent of 
232 context duration. This result was obtained for both a strongly incoherent context

233 consisting in acoustic syllables dubbed on a completely different video material extracted

234 from sequences of unscripted sentences, and for a phonetically incoherent context

235 obtained by dubbing audio syllables on video syllables having a different phonetic value,

236 while maintaining audiovisual synchrony.

237 It remains to be established what happens for smaller context durations. This is the 238 objective of the first experiment in which we will assess the role of short incoherent 239 contexts, from 0 to 3 seconds, to see what is the minimal duration of incoherence 240 necessary for providing significant unbinding (as displayed by a significant decrease in 241 the amount of the McGurk effect) and when does maximal unbinding occur.

\section{2. Conditions for rebinding after unbinding}

243 Supposing that the decrease in the McGurk effect produced by an incoherent audiovisual 244 contextual stimulus is indeed due to an unbinding mechanism, a question is to know 245 what kind of information is able to reset the system and put it back in its supposedly 246 bound default state.

247 The objective of the second experiment in the present paper is to attempt to answer this 248 question. For this aim, we will test whether applying a reset period of either coherent 249 material or silence after the incoherent unbinding context would enable to recover the 250 McGurk effect. The driving hypothesis of this experiment is the following: (1) the 251 incoherent context alone should decrease the McGurk effect and hence increase the 252 amount of "ba" responses; (2) the additional reset context, if it is efficient for rebinding, 253 should result in recovering the McGurk effect (possibly with a cumulative effect, that is 254 the amount of McGurk responses should increase for increasing durations of the reset 255 stimulus, back to its initial value without context when reset is long enough). 


\section{Experiment 1: Time constant of the unbinding process}

258 The first experiment aimed at estimating whether short incoherent audiovisual contexts

259 could indeed modulate the McGurk effect and at assessing the role of context duration in

260 the range corresponding to 0 to 3 seconds of incoherence. The paradigm was quite 261 similar to the one used in Nahorna et al. (2012), consisting in online monitoring of 262 congruent and incongruent McGurk targets embedded in a coherent or incoherent 263 context. The general hypothesis was that incoherent contexts should decrease the amount 264 of fusion responses "da" to McGurk targets, the experimental question being to know 265 how this decrease would depend on context duration. Response times, which are seldom 266 studied in audiovisual perception experiments, were also analyzed to assess how they 267 would depend on the target and context.

\section{A. Materials and Methods}

\section{Participants}

20 subjects, French native speakers without any reported history of hearing disorders and

271 with normal or corrected-to-normal vision participated in the experiment (4 women and 27216 men, from 23 to 54 years old with mean 26.6, 19 right-handed and 1 left-handed).

273 They all gave informed consent to participate in the experiment and were not aware of 274 the purpose of the experiments.

\section{2. Stimuli}

276 Subjects were presented with audiovisual films consisting of an initial part called context 277 followed by a second part called target (Figure 1). All stimuli were prepared from 
278 audiovisual material produced by a French male speaker, JLS, with lips painted in blue

279 to allow precise video analysis of lip movements (Lallouache, 1990). The videos 280 consisted of the entire speaker's face, keeping natural colors apart from the blue make-up.

281 Recordings were digitized at an acoustic sampling frequency of $44.1 \mathrm{kHz}$ and a video 282 sampling frequency of $50 \mathrm{~Hz}$ (25 images per second with two frames per image). All the 283 stimuli that will be described here under are exactly the same as those in Nahorna et al. 284 (2012), apart from smaller context durations in the present experiment compared with 285 Experiments 1 and 2 in Nahorna et al. (2012).

286 The target was either a congruent audiovisual "ba" syllable, or an incongruent McGurk 287 stimulus with an audio "ba" dubbed on a video "ga". To prepare incongruent "McGurk" 288 stimuli, the auditory channel of videos finishing with a "ga" was edited by replacing the 289 "ga" sound with a "ba" excerpt extracted from appropriate acoustic files. The "ba" sound 290 was positioned exactly at the same temporal position as the "ga" sound. Synchronization 291 was ensured by superposing temporal positions of the plosive burst at the onset of the 292 target stimulus. Congruent audiovisual "ba" syllables should be perceived as "ba", while 293 incongruent McGurk stimuli should often be perceived as "da" (McGurk and 294 MacDonald, 1976). The focus was actually on McGurk targets and the congruent "ba" 295 targets were only presented as controls.

296 There were three types of contexts in this experiment. The first type was coherent. It 297 consisted in a series of 1 to 5 audiovisual syllables extracted from random sequences

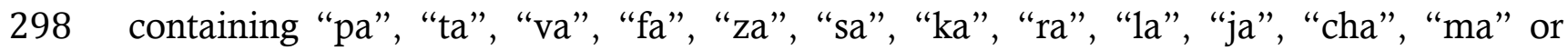
299 "na". The speaker was instructed to produce a short silence between consecutive 300 syllables, which was necessary for further audio editing. The syllable rhythm was about $3011.5 \mathrm{~Hz}$, hence the context duration varied between 0.6 and $3 \mathrm{~s}$ depending on the number 302 of uttered syllables. 
303 The second type was called strongly incoherent. This context consisted of either 1,2,3,4

304 or 5 acoustic syllables dubbed on an equally long stretch of a video of a speaker saying sentences.

306 The third type was called phonetically incoherent. It was obtained by swapping the audio content from one syllable to the other - keeping exactly the same video material as in the coherent context condition - while maintaining a precise synchrony in time between the auditory and visible syllables, hence the term phonetically incoherent. To maximize 310 audio-visual incoherence, syllables were firstly organized in five groups known to be 311 visually rather distinguishable (visemes): "pa, ma", "fa, va", "ta, na, sa, za", "cha, ja" 312 and "ka, la, ra, ga". Then the audio content of each syllable was swapped with the 313 content of a syllable from a different group. For each syllable, care was taken to maintain 314 perfect synchrony between the sound and the image by dubbing the sound with the burst 315 onset at exactly the same position as the original sound. Again, context duration was varied, such that the context consisted of either $1,2,3,4$ or 5 audiovisual syllables.

317 As recalled in Section I.C.1, both sets of incoherent contexts have already been shown in 318 Nahorna et al. (2012) to produce a significant decrease in the McGurk effect for context 319 durations larger than 5 syllables (typically 3 seconds). Therefore the question in 320 Experiment 1 is to know what happens for smaller durations.

321 A fixed set of target stimuli (comprising "ba" and "McGurk" stimuli) was used all along 322 the experiment. McGurk stimuli were presented three times more than congruent stimuli, 323 which served as controls. There were 4 different "ba" targets and 12 different McGurk 324 targets, positioned at the end of each of the three sets of context sequences and for each of 325 the 5 context durations (all 12 McGurk tokens and 4 ba targets were used equally often in 326 each condition). To ensure continuity between the end of the context stimulus and the 
onset of the target stimulus, a 200-ms transition stimulus (5 images without sound) was inserted between context and target (with a progressive linear shift from face to black from images 1 to 3 , and a progressive linear shift from black to face from images 3 to 5). Fading is a "necessary evil" to be able to carefully control both contexts and targets, hence finding a way to stick together these two pieces of audiovisual material. It could potentially predict the occurrence of targets, but does so then for all conditions. This would in fact provide some reset ingredient potentially decreasing the role of incoherent contexts, hence we cannot dismiss the assumption that incoherence effects could be underestimated because of a possible resetting effect due to fading. Subjects however never complained that there was a perturbing discontinuity from context to target, discontinuity actually being very difficult to notice thanks to the dubbing procedure described above $\mathrm{e}^{(2)}$.

An additional set of stimuli consisting in targets without context (4 "ba" and 12 McGurk targets) was also presented. These stimuli, introduced to provide a kind of reference for evaluation of the role of context, were not contained in the experimental plan (with three contexts and five context durations) hence they had a special status in the statistical analyses (see later).

This provides altogether 256 stimuli: 3 contexts * 5 durations * $(12$ McGurk targets +4 "ba" targets $)+(12$ McGurk targets +4 "ba" targets $)$ without context. The 256 stimuli were concatenated into a single film, with a $840-\mathrm{ms}$ inter-stimulus silent interval. The video component of this silent interval was made of the repetition of the last image of the previous stimulus. Such a short inter-stimulus interval was selected to put the subjects in a real monitoring task where there was large uncertainty about the temporal arrival of possible targets, to decrease as much as possible post-decision biases on target detection. A film was hence made of a random succession of coherent and incoherent contexts at all durations, and of targets without context (this was not a context-blocked experiment). All 
352 acoustic files were globally normalized in intensity to ensure that they were presented at

353 the same level. We prepared 5 different films with 5 different orders of the 256 stimuli

354 (each film lasted about 15 minutes). Each subject was presented with one film, the 5 films

355 being equally distributed between the 20 subjects ( 4 subjects per film).

\section{Context}

\section{Without context}

\section{Coherent}

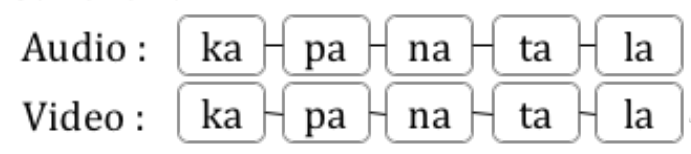

\section{Strongly incoherent}

Audio : ka pa na ta la

Video : Random sentence

\section{Congruent}

ba : Audio
ba : Video

\section{McGurk}

ba : Audio

ga : Video

\section{Phonetically incoherent}

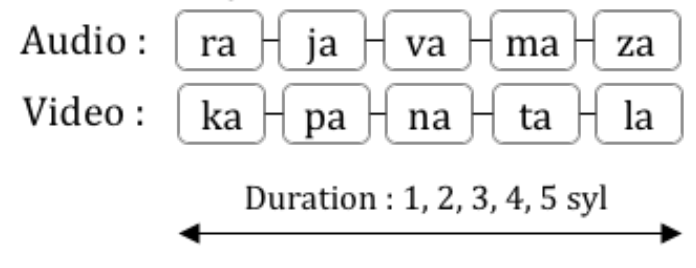

\section{Procedure}

361 The subject's task was to detect online "ba" or "da" syllables (syllable monitoring task),

362 without knowing when they could occur in the sequence. The experiment consisted of syllable monitoring with two possible responses - "ba" or "da" (responses entered on a

364 keyboard, with one button for "ba" and one for "da", the order of buttons being equally 
365 distributed across subjects). Therefore, subjects could provide responses at any time along

366 the monitoring process.

367 The experiment was monitored by the Presentation ${ }^{\circledR}$ software (Version 0.70,

368 www.neurobs.com). It was carried out in a soundproof booth with the sound presented 369 through an earphone at a fixed level for all subjects, the level being adjusted to be 370 comfortable for the task (around $60 \mathrm{~dB}$ Sound Pressure Level). The video stream was 371 displayed on a screen at a rate of 25 images per second, the subject being positioned at 372 about $50 \mathrm{~cm}$ from the screen. Instructions were to constantly look at the screen, and each 373 time a "ba" or a "da" was perceived, to immediately press the corresponding button 374 (displayed by the experimenter at the beginning of the experiment).

\section{Processing of responses}

376 The number of "ba" and "da" responses to the targets was computed for each subject and 377 each condition. Since the task was syllable monitoring and the subjects did not know 378 when the targets would occur, they could detect "ba" or "da" at any time and also fail to 379 detect the target (failures either due to lack of response or multiple different responses to 380 the target stimulus).

381 Analysis of response times enabled us to specify a protocol in which only responses 382 within $1200 \mathrm{~ms}$ after the target syllable acoustic onset were considered (target onset was 383 manually detected with the support of the MATLAB 7.6.0 software). This choice was 384 constrained by the short inter-stimulus interval (840 ms): $1200 \mathrm{~ms}$ after the burst onset of 385 the target stimulus was typically the onset time of the next stimulus. Furthermore, 386 responses intervening less than $200 \mathrm{~ms}$ after the burst were also discarded (see e.g. 387 Ratcliff \& Rouder, 1998; van Maanen et al., 2012). In the case of two different responses 388 inside this [200-1200] window, the responses were discarded. Altogether (that is adding 
389

390

391

392

393

394

395

396

397

the number of misses or different responses to the target), this resulted in a total of $8.9 \%$ of cases with no response to a target stimulus. This amount is not surprising considering that the subjects only had two possible answers at their disposal while McGurk stimuli could result in percepts other than "ba" and "da" in French (Cathiard et al., 2001), and that they had less than $1.2 \mathrm{~s}$ to answer online. The number of no-response was actually larger for McGurk than for "ba" targets. Importantly, the amount of cases with no response was rather stable for McGurk targets across the three context conditions, varying between 9.3 and 11\%, hence this protocol did not bias the following analyses.

Response time was defined as the time separating the plosive burst at the onset of the target stimulus and the response (within the $1200 \mathrm{~ms}$ cutoff) measured with the Presentation ${ }^{\circledR}$ software. For each (subject, target, context, duration) condition, the mean response time was estimated by averaging the response times for all stimuli in the corresponding condition.

\section{Statistical analyses}

Considering responses, analyses were performed on proportions of "ba" responses over the total number of "ba" plus "da" responses (ignoring cases where no response was provided by the subjects), after processing them with an asin(sqrt) transform to ensure quasi-Gaussian distribution of the variables involved. A systematic check was made that other analyses performed either on the proportions of "ba" responses over the total number of stimuli ("ba" plus "da" plus no response) or on the proportions of "da" responses over the total number of stimuli provided the same significant and nonsignificant effects. Since "ba" targets were only there as controls, the analysis of responses was focused on McGurk targets. 
412 To quantitatively assess the comparative role of the three contexts and their five

413 durations, a repeated-measures ANOVA was done on transformed proportions of "ba"

414 responses for McGurk targets, with context (3 values) and context duration (5 values) as

415 independent variables and subject as a random-effect factor. Greenhouse-Geisser

416 correction was applied in case of violation of the sphericity assumption. When

417 appropriate, we used post-hoc analyses of differences between two conditions with

418 Bonferroni corrections, and reported differences as significant in case of a Bonferroni-

419 corrected value $\mathrm{p}<0.05$. Importantly, the data for targets without context were not

420 considered in the ANOVA since they are not part of the experimental plan with 3

421 contexts and 5 context durations. However, since they were recorded to provide a

422 reference, specific t-tests comparing the context conditions to this no-context condition

423 have been conducted following the results of the ANOVA.

424 Considering mean response times per subject and condition, a repeated-measures

425 ANOVA was performed on the logarithm of these values for ensuring normality of the

426 distributions, with the same independent variables as previously. A repeated-measures

427 ANOVA was done on logarithms of mean response times with target (2 values), context

428 (3 values) and context duration (5 values) as independent variables and subject as a

429 random-effect factor. Once again, the no-context condition was not introduced in these

430 ANOVAs and rather played the role of a baseline for evaluating the role of context. 


\section{B. Results}

\section{Effect of context on the amount of "ba" responses}

437 The results of the subjects' responses (proportion of "ba" responses relative to the total 438 number of "ba" + "da" responses) for both targets in the three contexts and without 439 context are set out in Figure 2. "ba" targets are classified as "ba" in all contexts with a 440 score close to $100 \%$ (varying between $98.3 \%$ and $99 \%$ in the three contexts). McGurk 441 targets produce a smaller proportion of "ba" responses, but this proportion is much larger 442 in the strongly incoherent and slightly larger in the phonetically incoherent contexts than 443 in the coherent context. The repeated-measures two-factor ANOVA on scores for

444 McGurk targets shows that the effect of context is indeed significant $[\mathrm{F}(2,38)=58.425$, $445 \mathrm{p}<0.001])$. Post-hoc analysis confirms that the differences between the three contexts are 446 significant. The increase in the proportion of "ba" responses to McGurk targets from the 447 coherent $(45 \%)$ to the strongly incoherent context $(73 \%)$ is very large and corresponds 448 actually to a reduction of the McGurk effect by half (from $55 \%$ of "da" responses with 449 coherent context to $27 \%$ with strongly incoherent context). The difference is much 450 smaller - though significant - with the phonetically incoherent context $(10 \%$ increase in 451 "ba" responses from 45\% to 55\%). Paired t-tests comparing either the target with 452 coherent context or the target with phonetically incoherent context to the reference 453 provided by the target without context provide no significant difference (without context 454 compared to coherent context: $55 \%$ vs. $45 \%,[\mathrm{t}(19)=1.54, \mathrm{p}>0.139]$; without context 455 compared to phonetically incoherent context: $55 \%$ vs. $55 \%,[t(19)=0.001, p=1])$. 
457

458

459

460

461

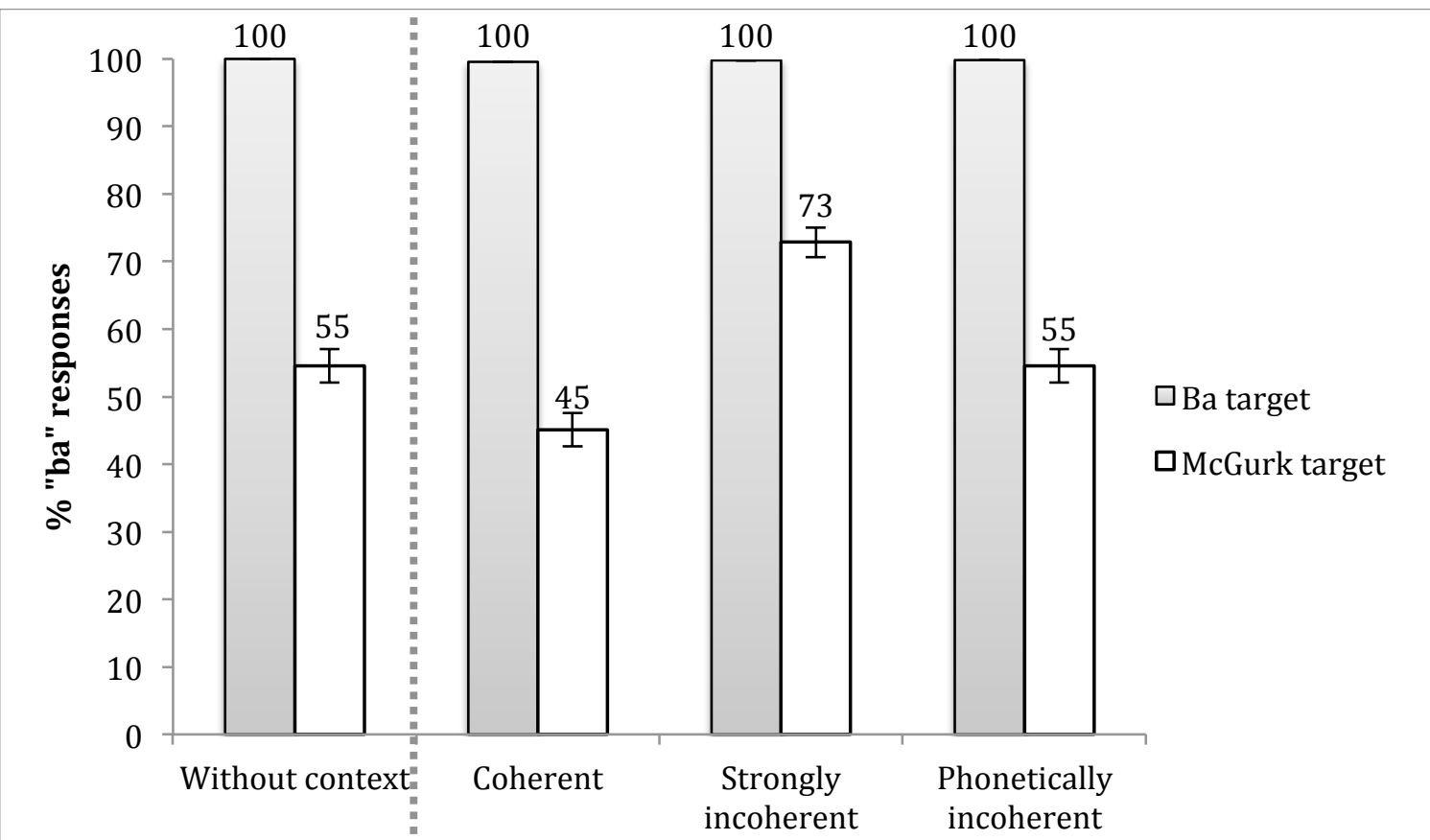

Figure 2 - Percentage of "ba" responses (relative to the total number of "ba" + "da" responses) for the two targets in the three contexts and without context.

\section{Effect of context duration}

463 Concerning durations, the ANOVA displays a main effect of the duration factor

$464[\mathrm{~F}(4,76)=5.44, \mathrm{p}<0.001]$ and a significant interaction with context $[\mathrm{F}(8,152)=3.558$, $465 \mathrm{p}<0.001]$ (Fig. 3). Post-hoc analyses show that the duration factor is significant only for 466 the strongly incoherent context. For this condition, the only significant differences are 467 between durations 1 or 2 syllables on one hand and 4 syllables on the other hand.

468 Globally, the trend for the strongly incoherent context is that the strong reduction of the 469 McGurk effect is not only quick, complete as soon as one syllable of incoherent context, 470 but even larger for the smallest context durations. We will propose possible 471 interpretations of this unexpected fact later in the discussion. Concerning the phonetically 472 incoherent context, since duration does not seem to matter, this suggests that the small 
reduction of the McGurk effect with this context compared with the coherent context is rapid and complete for a one-syllable duration, as for the other incoherent context.

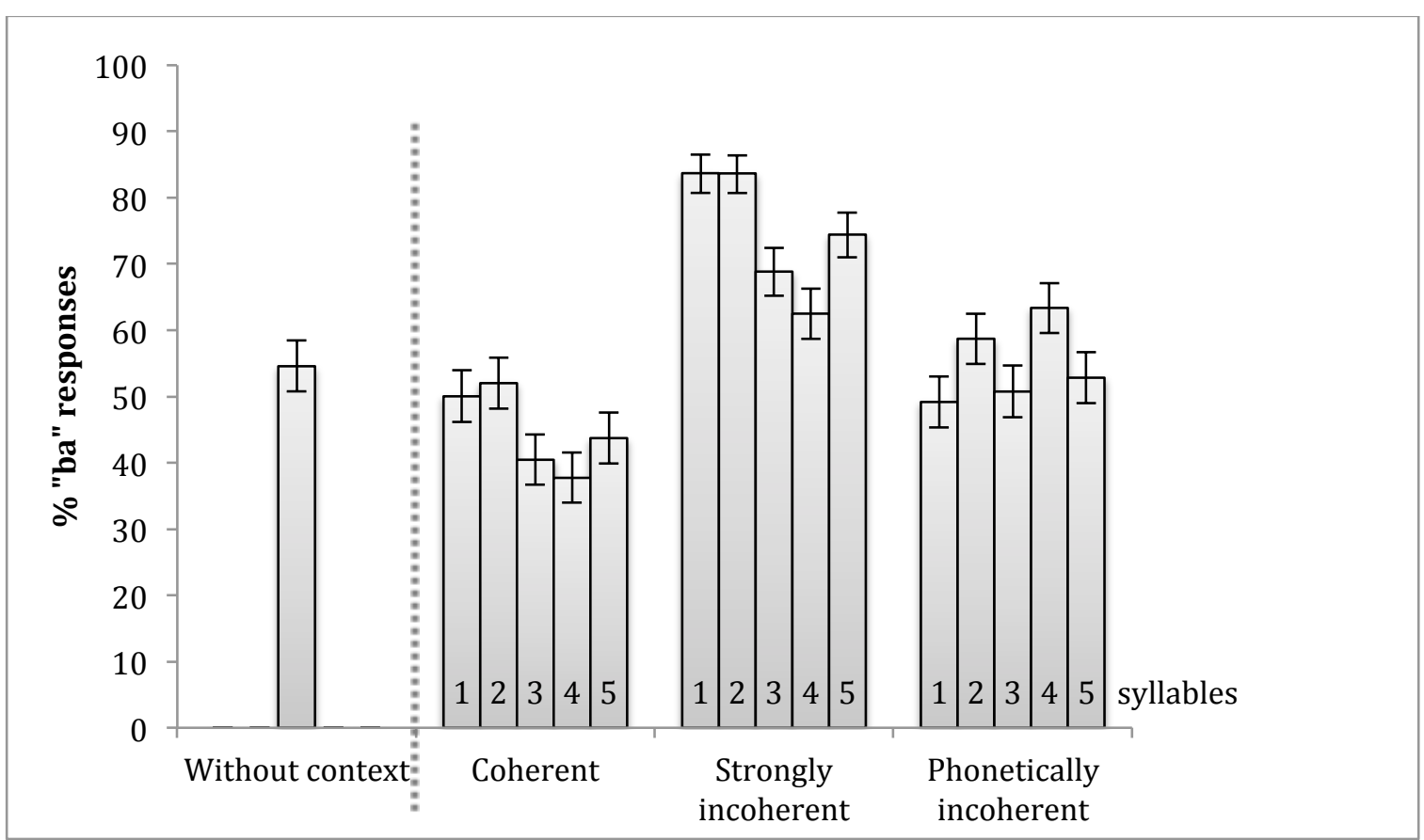

Figure 3 - Percentage of "ba" responses for McGurk targets for the three contexts and their five durations, compared to targets without context.

\section{Contextual effects provided by previous stimuli}

A possible problem in the previous analyses concerns the possibility that the response to a given stimulus may be influenced by the previous stimulus. This would produce possible spillover effects, e.g. the no-context condition would in fact be influenced by the previous coherent or incoherent contexts; or the coherent context condition would be contaminated by a previous stimulus with an incoherent context, etc. This question was already discussed in our previous study (Nahorna et al., 2012), and we will provide the same kind of analyses to evaluate this question. Firstly we performed a new repeated- 
490

491

492

493

494

495

496

497 511 categorizes a given McGurk stimulus as "ba" (respectively "da"), there is an increased 512 chance that the next McGurk stimulus will stay perceived as "ba" (respectively "da").

513 Contrast effects appear when the response to a stimulus in a given category C1

measures ANOVA on global scores (all context durations together) for McGurk targets, with three factors: subject (random), context and preceding context (fixed). Notice that although the set of target stimuli is of course the same from one context to the other, it is not controlled for being the same from one previous context to the other, which makes this analysis arguable. It appears that both the effects of context $[F(2,38)=51.192$, $\mathrm{p}<0.001]$ and preceding context $[\mathrm{F}(2,38)=4.252, \mathrm{p}=0.022]$ are significant, but not their interaction $[\mathrm{F}(4,76)=0.335, \mathrm{p}=0.854]$. The significant effect of context corresponds to the results presented previously (see Section II.B.1 and Fig. 2). The significant effect of preceding context suggests that it plays a role in the binding and decision process, with a mean $5.5 \%$ increase in "ba" responses (averaged over all McGurk targets for the three contexts) from a preceding context which is coherent to a preceding context which is strongly incoherent. The lack of significant interaction means that the effect of preceding context is the same for all current contexts.

However, we reasoned in Nahorna et al. (2012) that another important bias could come not from the previous stimulus but from the previous response. Indeed, if the preceding context is strongly incoherent, the preceding response to McGurk targets is more often a "ba". Might this play a role in the decision for the next McGurk target? Actually, this should be the case, considering two classical response biases that are recalibration and contrast (Bertelson et al., 2003; Vroomen and Baart, 2011). Recalibration effects appear when subjects modify their categories - and hence their decisions - in relation with the decision they took for previous stimuli. The possibility here would be that when a subject Contrast effects appear when the response to a stimulus in a given category $\mathrm{Cl}$ 
514 (contrasted to another category $\mathrm{C} 2$ ) is more likely to be " $\mathrm{C} 1$ " if the preceding stimulus

515 was in category C2 than if it was in category C1.

516 These two kinds of effects were indeed clearly displayed in the data analyzed by Nahorna

517 et al. (2012). The same phenomenon appears in the present study, as can be seen on

518 Fig. 4 where we report the scores for McGurk targets depending on context, preceding

519 context and preceding response (incoherent context in this figure is the strongly

520 incoherent context: we do not present results for phonetically incoherent context to make

521 the figure clearer). On this figure, we observe the difference between the coherent and

522 incoherent contexts with more "ba" responses in the second case (the "ba" score

523 increases when comparing the first set of 3 bars with the second one, or the third one with

524 the fourth one). However, there is in each case a large modulation depending on the 525 preceding stimulus and response. Indeed, for each set of 3 bars (that is for each 526 configuration of precedent context and present context) there is a recalibration effect with 527 a much larger "ba" score when the precedent target was a McGurk target with "ba" 528 response, compared with the "ba" score when the precedent target was a McGurk target 529 with "da" response. There is also probably a contrast effect with a decrease in "ba" 530 responses when the previous target was a "ba" compared to when it was a McGurk target 531 with "ba" response - though it is not easy to disentangle contrast from recalibration.

532 Of course, since the preceding context modifies the amount of "ba" responses to the 533 McGurk targets, the induced response biases may explain the effect of preceding context 534 displayed in the ANOVA. Actually, the size of recalibration effects $(50 \%$ or more in Fig. 5354 ) is much larger than the size of the global effect due to the preceding context. Once the 536 previous decision is taken into account, if we compare the first set of three bars with the 537 third one or the second one with the fourth one in Fig. 4, we notice that in most cases the 538 amount of "ba" responses is in fact higher when the preceding context is coherent 
539 compared with when it is incoherent. Therefore altogether, we may consider that the

540 present results are not contaminated - or at most very weakly - by the context of a

541 previous stimulus, though they are subject to classical contrast and recalibration

542 phenomena providing some decision biases. It might appear surprising that context

543 effects are more or less restricted to one target and seem more or less "reset" when the

544 next stimulus is presented: we will come back on this point in the General Discussion

545 (Section IV.3).

546

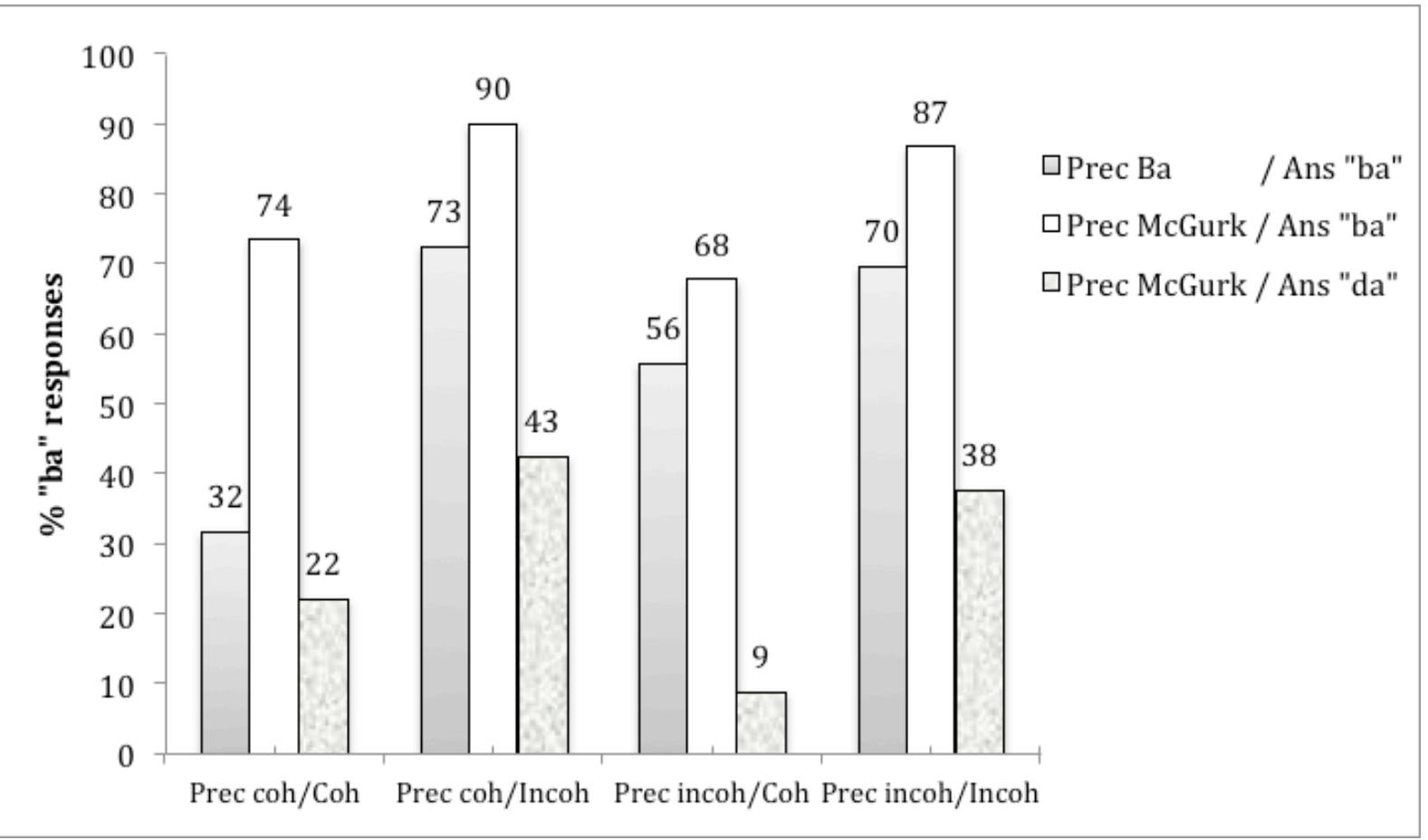

548 Figure 4 - Effect of the preceding decision in Experiment 1. Responses to McGurk

549 stimuli depending on context ("Coh" for coherent, "Incoh" for incoherent), preceding

550 context ("Prec coh" for coherent preceding context, "Prec incoh" for incoherent 551 preceding context), preceding target stimulus ("Prec ba" vs "Prec McGurk") and 552 previous answer ("Ans ba" for previous "ba" target, "Ans ba" and "Ans da" for previous 
553 "McGurk" target). Incoherent context in this figure is the strongly incoherent context: we

554 do not present results for phonetically incoherent context to make the figure clearer.

555

\section{4. Analysis of response times}

557 Mean response times for both targets in the three contexts and without context are set out

558 in Figure 5. Response times appear to be globally larger without context, and not

559 different from one context to the other. Response times are also systematically larger for

560 McGurk targets. These trends are confirmed by the three-way ANOVA. There is a

561 significant effect of target $[F(1,19)=28.52, \mathrm{p}<0.001]$, with a $58.3 \mathrm{~ms}$ difference between

562 mean response times for "ba" and McGurk targets. There is no effect of context, either

563 alone or in interaction with any other factor.

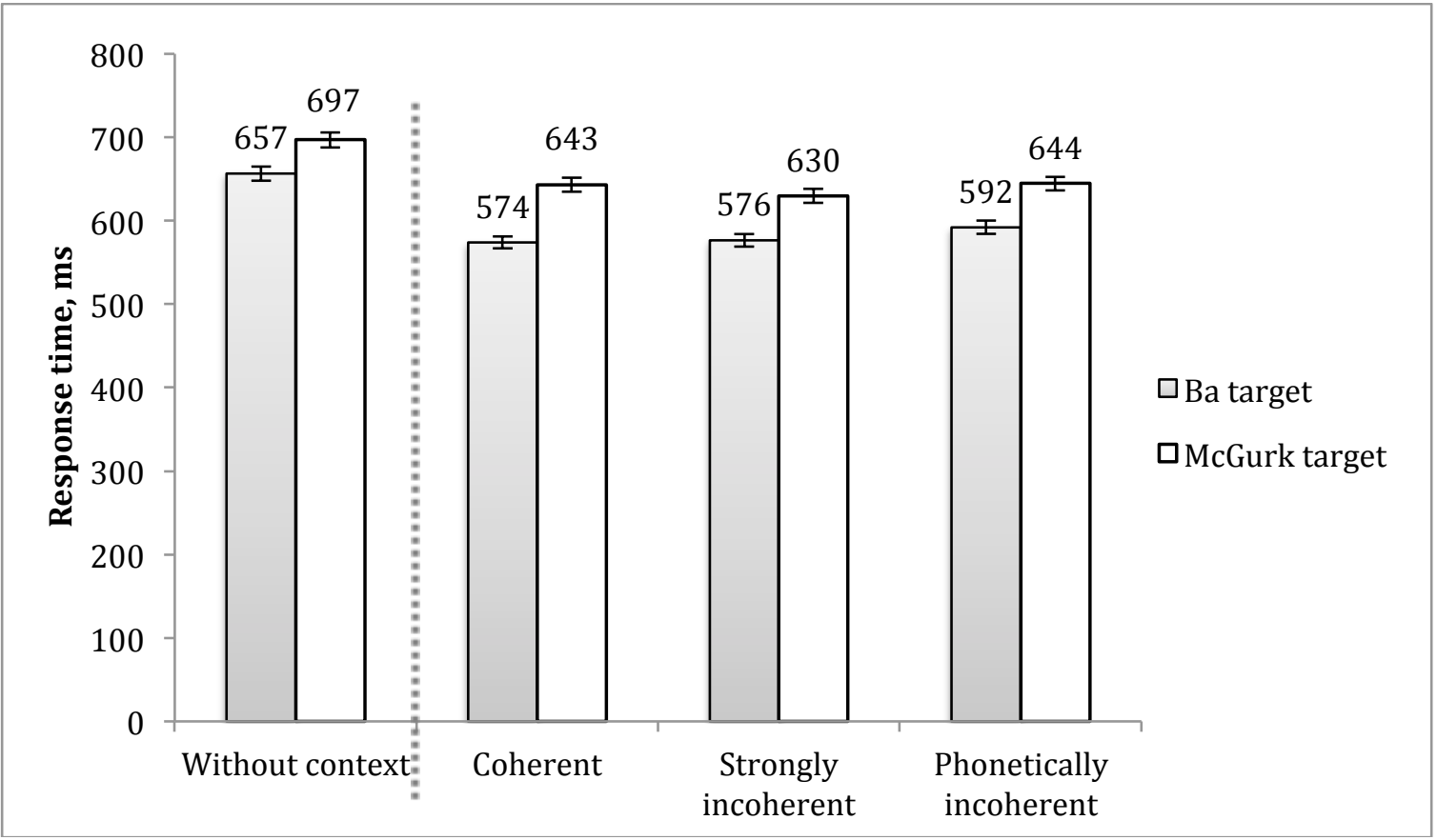


569 There is also a significant effect of context duration $[\mathrm{F}(4,76)=3.41, \mathrm{p}<0.03]$ and of the

570 interaction between target and context duration $[\mathrm{F}(4,76)=4.16, \mathrm{p}<0.004]$. The effect of

571 duration is displayed in Figure 6. It appears a global trend in which response time

572 decreases with context duration, from no context to 5 syllables. Post-hoc analyses display

573 significant differences between response times (averaged over "ba" and McGurk targets)

574 at 1 vs. 2 and 3 syllables. The effect of duration could be due to the fact that context

575 enables the subjects to prepare the arrival of the target stimulus and hence respond more

576 quickly when it finally arrives. This could explain the trend for having larger response

577 times without context (Figure 5).

578

579

580

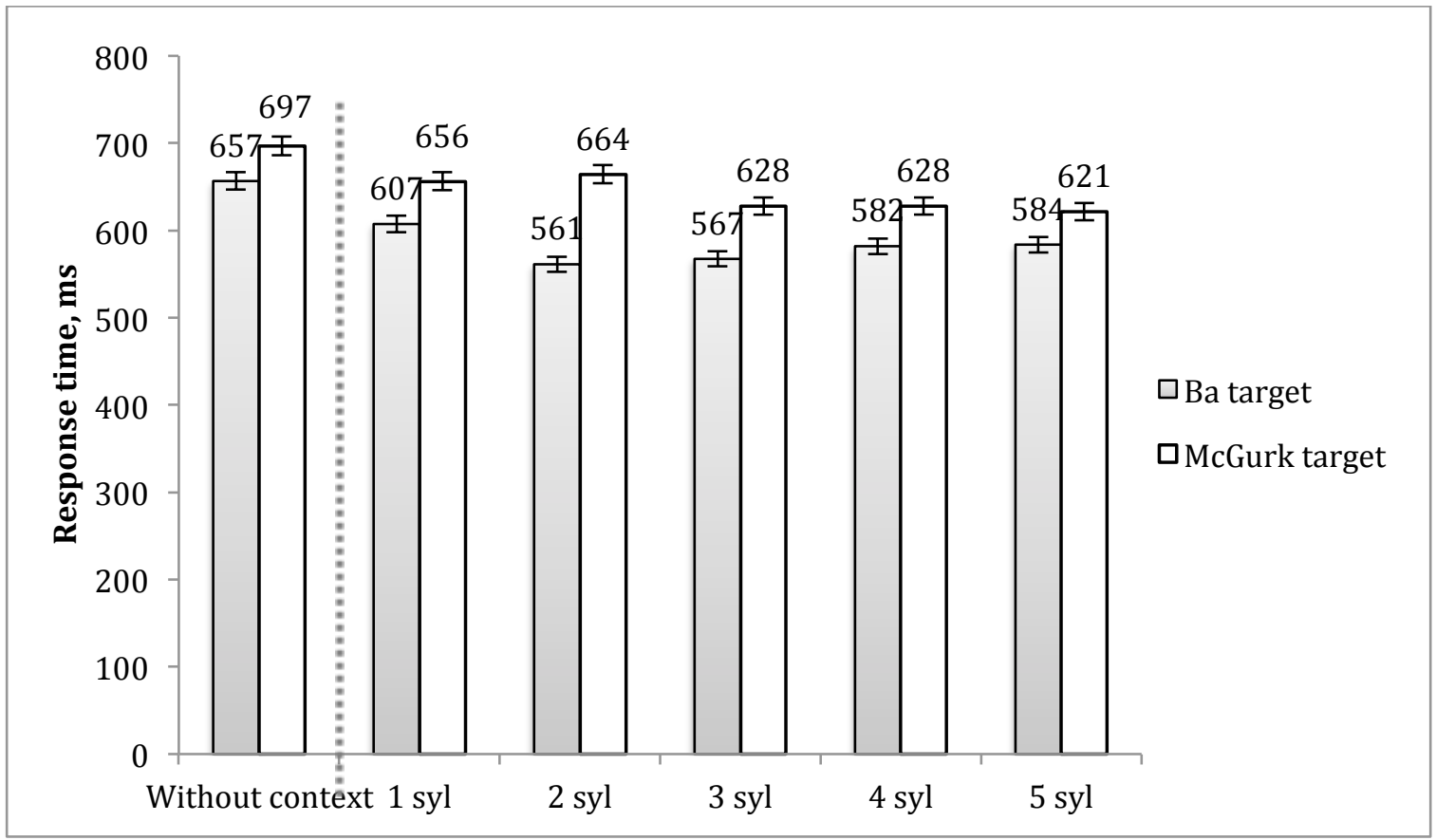

581

Figure 6 - Mean response times for the two targets

582 in the five context durations and without context. 


\section{Discussion}

587 Four major facts emerge from this experiment. Firstly, the present data confirm those

588 obtained in the princeps study by Nahorna et al. (2012): various kinds of incoherent 589 audiovisual contexts decrease the strength of the McGurk effect and increase the amount 590 of auditory responses to McGurk targets. For strongly incoherent contexts the size of the 591 reduction in the McGurk effect is similar in the present data and in the previous ones by

592 Nahorna et al. (2012): typically a reduction by half. For phonetically incoherent contexts 593 the size is much smaller, though significant: while there was also a reduction of the 594 McGurk effect by half compared with the coherent context in the princeps paper (see 595 Experiment 2 in Nahorna et al., 2012) it is much smaller here (55\% ba" responses with 596 phonetically incoherent context vs. $45 \%$ for coherent context, see Figure 2 ). This is likely 597 due to the fact that both incoherent contexts were presented in the same experiment here 598 while they were studied in two separate experiments in the previous study. This seems to 599 have induced a kind of calibration process for subjects of the present study, in which the 600 size of incoherence is compared from one stimulus to another. However the present data 601 confirm that pure phonetic audiovisual incoherence keeping a perfect audiovisual 602 synchrony allows some amount of unbinding between sound and image when compared 603 with coherent context. But they show that this is only a small part of the total amount of 604 incoherence available in the strongly incoherent context: hence the corresponding 605 amount of decrease in the McGurk effect is much smaller for pure phonetic incoherence.

606 Secondly, we now have a clear confirmation that the unbinding effect is rapid. One 607 syllable seems to suffice to produce an effect as large as the effect of five syllables - and in 
608 Nahorna et al. (2012) there was no difference between 5 and 20 syllables. Hence it seems

609 that unbinding is almost complete with a small duration of incoherence (around $600 \mathrm{~ms}$ ),

610 typically one syllable. This appears to be the case for both contexts. For the strongly

611 incoherent context, there is even a trend that small durations ( 1 or 2 syllables) produce a

612 larger decrease in the McGurk effect than larger ones (4 syllables). This is rather

613 counterintuitive. It could be due to non-monotonous contrast effects in the computation

614 of audiovisual coherence (with a kind of incoherence adaptation effect that would

615 increase the size of perceived incoherence at the first time when some incoherence is

616 perceived). It could also be related with the increase in response times for short contexts

617 compared to longer ones (see Figure 6). Indeed, this could be taken as an indicator that

618 the subject is surprised by the arrival of the target for short contexts, and that surprise

619 could lead to decreased fusion, considering the audiovisual integration has been shown to

620 falter under high attention demands (Alsius et al., 2005).

621 The third point concerns the nature of the default state. Our hypothesis was that without 622 context subjects would be in a default state of binding. The mere fact that the McGurk 623 effect exists shows that there is indeed a certain amount of binding without context. It 624 remains to be known if binding is maximal in the default state The fact that there is no 625 significant difference between the no-context and coherent context conditions and no 626 effect of context duration for the coherent context condition suggests that this might be 627 the case. This is further supported by the results from our previous study, where we found 628 no effect of context duration from 5 to 20 syllables. However, since the phonetically 629 incoherent context also displays no significant difference with the no-context condition, 630 we cannot dismiss the possibility that there would be in fact no unbinding effect of the 631 phonetically incoherent context compared with the no context condition (the default 632 state) and some increase of the amount of binding when a coherent context is applied to 
633 the default state. The (non significant) $10 \%$ decrease of the "ba" percentage from the no-

634 context condition to the coherent context condition (see Fig. 2), together with the (non 635 significant) decrease trend of the "ba" score in the coherent context when context 636 duration increases from 1 to 5 syllables (see Fig. 3) might call for further experiments to 637 test this assumption. Let us conclude, to summarize the discussion of this third point, 638 that the default state (without context), which we will still consider as "bound" since it 639 displays a certain amount of audiovisual integration, is perhaps not maximally bound; 640 and that the possible increase in binding that could be produced by a coherent context, if 641 it exists, does not seem very large.

642 The last important finding in Experiment 1 is that response times are consistently larger 643 for McGurk targets than for congruent "ba" targets independently on the effects of 644 context (Figure 5). This is rather striking considering the size of context effects on the 645 scores of "ba" responses. Indeed, it is classically considered that response times in such 646 experiments rely heavily on the ambiguity of the stimulus to process (Massaro and 647 Cohen, 2003). In the present case, the ambiguity in McGurk targets is largely reduced by 648 the very incoherent context: while these targets are identified close to $50 \%$ as "ba" 649 (actually 45\% "ba" vs. 55\% "da") in the coherent context, they are perceived as $73 \%$ as 650 "ba" in the very incoherent context (see Figure 2). However this does not result in any 651 significant change in response times: context seems to modify the response but not the 652 response time. This suggests that the increase in response times for McGurk stimuli is 653 due, at least partly, to the detection of a local audiovisual incoherence, which seems to 654 slower the response independently on the response itself. We will come back to this point 655 in the general discussion. 


\section{III. Experiment 2: Testing the existence of a rebinding}

658 process

659 The results of Experiment 1 clearly show that an incoherent context results in a decrease

660 of the McGurk effect, which is due in our interpretation to an unbinding mechanism. The

661 objective of Experiment 2 is to know what kind of information is able to reset the system

662 and put it back in its bound default state (recalling the previous discussion about the fact 663 that the default state is not necessarily "maximally bound"), that is enhance the McGurk 664 effect again so that it recovers the level it has with no contextual stimulus before the 665 McGurk target.

666

667 A. Materials and Methods

668 1. Participants

66920 French subjects without hearing or vision problems participated in the experiment (9

670 women and 11 men, from 18 to 60 years old, mean 25.7, 19 right-handed and 1 left-

671 handed). They all gave informed consent to participate in the experiment, and were not 672 aware of the purpose of the experiments.

\section{2. Stimuli}

674 The stimuli, described in Figure 7, consisted in a succession of three components (with a 675 5-images fading between consecutive stimuli as in Experiment 1):

676 - A context which could be either coherent or "strongly incoherent" in the sense of Experiment 1. Therefore we discarded phonetically incoherent context in this 
experiment, to focus on the two most extreme variants that are coherent and strongly incoherent. In the following of Experiment 2, incoherent will refer to the strongly incoherent type of context. Considering the results of Experiment 1 showing no influence of context duration for coherent context, and a small significant difference between small ( 1 or 2 syllables) and large (4 syllables) durations for strongly incoherent contexts, we used only 2-syllable and 4-syllable durations;

a reset stimulus consisting in either $0,1,2$ or 3 coherent audiovisual syllables (coherent reset) or audio silence with fixed image of duration 0, 480, 1000, $1480 \mathrm{~ms}$ corresponding roughly to the same duration as the $0-, 1-, 2$ - or 3-syllable coherent reset condition (fixed reset). The reset was inserted only after incoherent contexts: coherent contexts were followed directly by the target, and used only as controls in this experiment. Notice that the "0-syllable reset" conditions actually mean no reset at all, and that these conditions are of course the same for both the coherent reset and the fixed reset, though it was necessary to introduce both conditions to ensure a full factorial design; audiovisual "ba" or a McGurk stimulus consisting in an audio "ba" dubbed on a video "ga". As in Experiment 1, McGurk targets were presented three times more than congruent "ba" targets, which served as controls.

698 Stimuli were presented to participants in two blocks, one block with coherent reset and 699 the other one with fixed reset. Each block comprised stimuli with either the coherent context (with 2 possible durations) with no reset, or the incoherent context ( 2 possible 701 durations) followed by the reset (4 possible durations). Hence there were altogether 10 702 conditions per block, with 4 different occurrences of a "ba" target and 12 different 
703

occurrences of a McGurk target per condition, with a total of 160 stimuli in a block,

704 presented in a random order and organized in a film as in Experiment 1, with the same 705 840-ms inter-stimulus interval. The order of blocks was randomized between the 20 706 subjects with 10 subjects per order.

707

Context

Incoherent

Audio ka-pa-na-ta

Video Random sentences

$\stackrel{\text { Duration : 2, } 4 \text { syl }}{\longrightarrow}$
Reset

(a) Coherent

(b) Fix image and silence

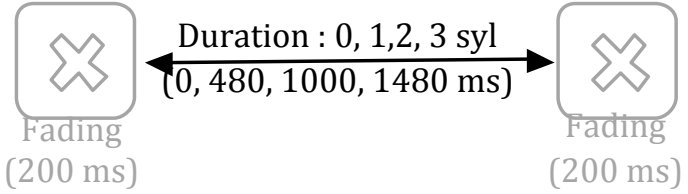

Target

\section{Congruent}

ba : Audio
ba : Video

McGurk

ba : Audio

ga : Video

Coherent - without reset

Audio ka pa na ta

Video ka pa na ta

708

Duration : 2, 4 syl

709

Figure 7 - Organization of stimuli in Experiment 2.

710

711 3. Procedure, processing of responses and statistical analyses

712 Procedure and response processing were exactly the same as in Experiment 1. The

713 number of missing responses in this experiment (still with the [200-1200 ms] cut off

714 procedure) was less than in Experiment 1 (7.6\%), Once again however, the amount of 715 cases with no response for McGurk targets was rather stable across the two reset 716 conditions, varying between 7 and 9.4\%. 
717 Statistical analyses were performed on the same variables as in Experiment 1: for each

718 subject and condition, proportions of "ba" responses over the total number of "ba" plus

719 "da" responses processed with an asin(sqrt) transform, and logarithm of mean response

720 times. Only the stimuli with incoherent context plus reset were submitted to repeated-

721 measures ANOVAs, the stimuli with coherent context without reset being only

722 considered as a baseline over which unbinding and rebinding were evaluated.

723

724 B. Results

725 1. Analysis of "ba" responses

726 As in Experiment 1, the "ba" target leads to $100 \%$ "ba" responses in both experiments 727 and in all conditions. Therefore, as planned, we will concentrate on McGurk targets. A 728 repeated-measures three-factors ANOVA on scores for McGurk targets with factors 729 context duration ( 2 vs. 4 syllables), reset type (fixed vs. coherent) and reset duration ( 0,1 , 7302 or 3 syllables) shows the following results.

731 The effect of context duration is significant $[F(1,19)=18.89, p<0.001]$. The shorter 732 context with 2 syllables produces in average a percentage of "ba" responses $5.4 \%$ larger 733 (that is a smaller McGurk effect) than the longer context with 4 syllables. There is no 734 interaction between context duration and any other variable, hence this effect is stable for 735 all reset conditions, whatever the reset type and duration.

736 The effects of reset type and reset duration are displayed on Figure 8. The effects of reset 737 type $[\mathrm{F}(1,19)=5.097, \mathrm{p}=0.036]$, reset duration $[F(3,57)=12.64, \mathrm{p}<0.001]$, and the 738 interaction between reset type and reset duration $[F(3,57)=11.699, \mathrm{p}<0.001]$ are all 739 significant. Actually, three major facts emerge from Figure 8. 
- Unbinding with incoherent context. Let us first look at what happens for the incoherent context without reset, corresponding to the 0-syl condition (left bars, for both types of resets). The score of "ba" responses is around $75-80 \%$, much larger than the score for the coherent context condition (rightmost bars), which is less than $50 \%$. This replicates the decrease of McGurk effect from coherent (more than $50 \%$ McGurk effect) to incoherent context (less than 25\% McGurk effect) displayed in Experiment 1. condition on Figure 8, it appears that this reset (made of acoustic silence + fixed image) provides almost no rebinding, since the "ba" score only slightly decreases from 0 to 1 -syl (that is $480 \mathrm{~ms}$ duration), then remains stable and stays much larger than the score for coherent context even for the longest reset duration (3-syl corresponding to $1480 \mathrm{~ms}$ ). Post-hoc analyses confirm the initial small decrease in "ba" responses, since there is a significant difference between scores at 0 and 2 syllables. However, a t-test confirms that the score at 3 syllables (74\%) is significantly different from the score with coherent context $(46 \%): t(19)=5.22$, $\mathrm{p}<0.001$

- Good rebinding with coherent reset. On the contrary, looking at the bars corresponding to the coherent reset condition, we observe that the "ba" score regularly decreases with reset duration and reaches the same value as for coherent context, coming back to its default state for the largest coherence period of 3 syllables. Post-hoc analyses confirm that the score at 0 is significantly higher than with 1, 2 or 3 syllables, and the score at 1 or 2 syllables is significantly higher than with 3 syllables. A t-test confirms that the score at 3 syllables $(43 \%)$ is not different from the score with coherent context $(45 \%)$ : $t(19)=0.624, p=0.54$. 


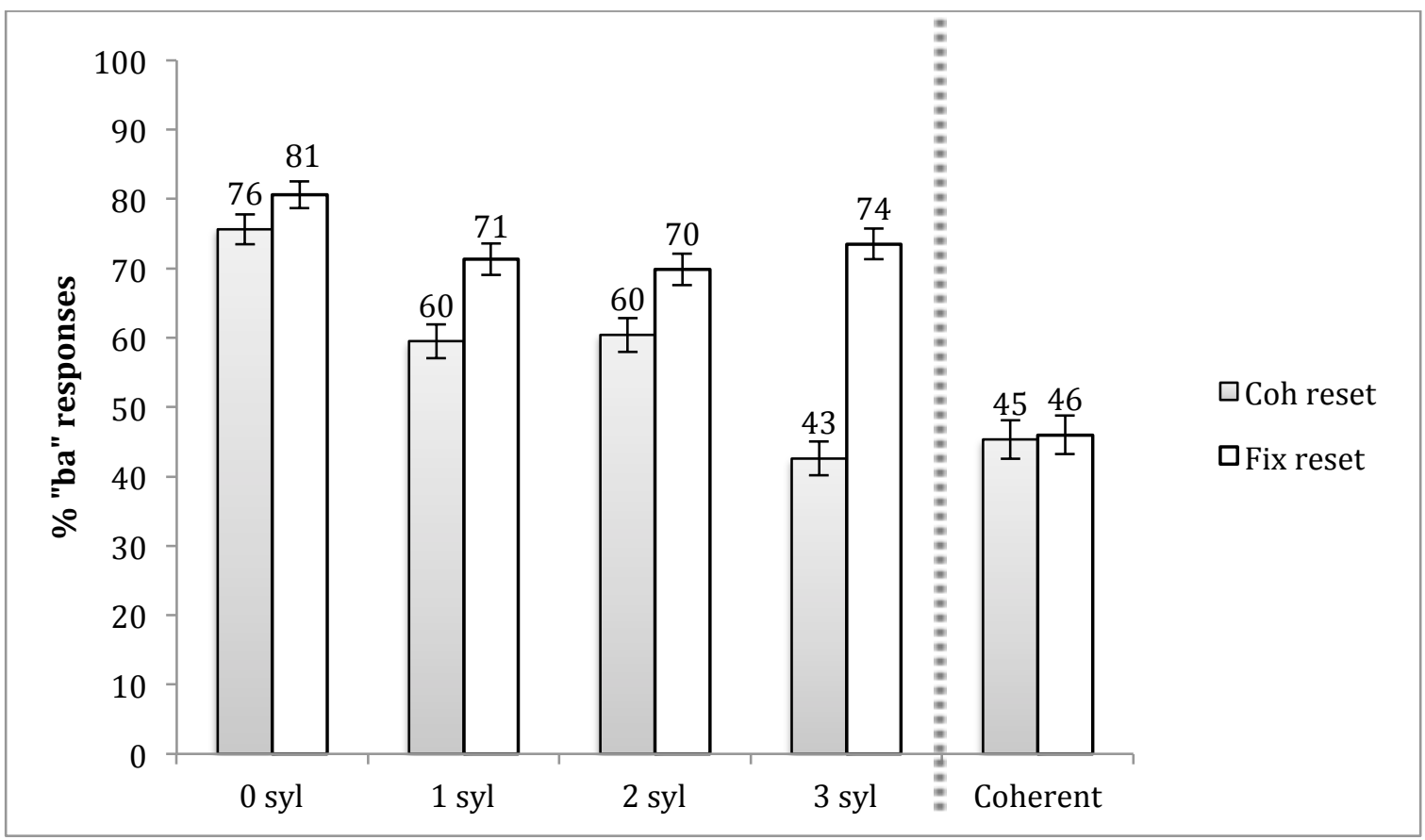

Figure 8 - Percentage of "ba" responses (relative to the total number of "ba" + "da" responses) for the McGurk targets with coherent context and with incoherent context for the two reset types and the four reset durations.

\section{Analysis of response times}

773 Mean response times for both targets in the two reset conditions are displayed in Figure

9. Response times are once again larger for McGurk targets. A two-way repeated-

775 measures ANOVA on target and reset type shows an effect of target $([\mathrm{F}(1,19)=29.57$,

$776 \mathrm{p}<0.001]$; difference between mean response times for "ba" and McGurk targets:

$77749.5 \mathrm{~ms}$ ) but no effect of reset, alone or in interaction with target. 


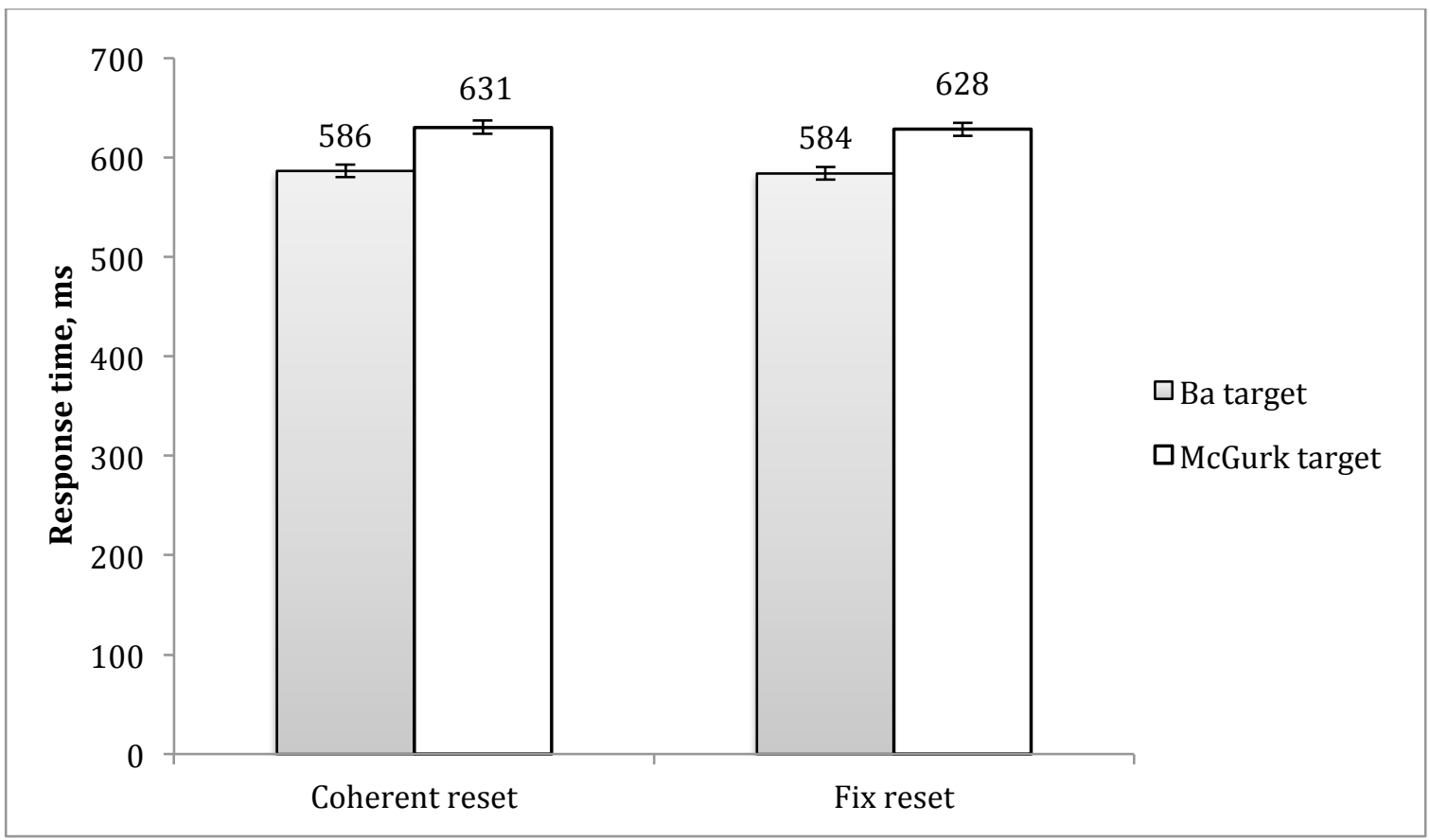

Figure 9 - Mean response times for the two targets in the two reset conditions.

\section{Discussion}

This experiment firstly confirms the amount of unbinding provided by the incoherent context (corresponding to the strongly incoherent context in Experiment 1), which produces a relative reduction of the McGurk effect by more than half. There is also a confirmation that short incoherent contexts ( 2 syllables) produce a larger decrease in the McGurk effect than longer ones (4 syllables), with a significant increase in the score of

791 "ba" responses around 5.4\% in the first case. The fact that this increase is not dependent on the reset duration (from 0 to 3 syllables) renders less plausible our interpretation in

793 Experiment 1 about the possible role of surprise since this should lead to differences 794 between short resets where the target comes rather quickly for the short context and long 795 resets where surprise is more unlikely. 
796 The major new result of Experiment 2 is that after an incoherent context decreasing the

797 McGurk effect, a coherent reset stimulus may increase it again until the original McGurk

798 level is recovered. However, while the decrease is rapid in Experiment 1 with a

799 maximum decrease already obtained for a one-syllable long context, the recovery appears

800 slower in Experiment 2, not complete before 3 coherent syllables are presented. On the

801 contrary, the other type of reset material composed of acoustic silence and fixed image 802 does not allow to recover the original McGurk effect: the level of McGurk responses after 803 a 2-syllable or 4-syllable period of incoherence remains remarkably stable at a low value 804 after a period of fixed reset up to $1.5 \mathrm{~s}$ (see Figure 8).

805 Finally, this experiment provides a confirmation concerning the pattern of response

806 times. Indeed, it appears (Figure 9) that response times are consistently longer for 807 McGurk targets than for congruent "ba" targets independently on the effects of reset.

808 This happens in spite of the strong effects of reset type and reset duration on the scores of 809 "ba" responses: reset modifies the response but not the response time. This confirms that 810 response times are not completely predictable from the ambiguity of the stimulus to 811 process.

812

\section{IV. General Discussion}

814 The two experiments presented in this paper confirm that context modulates the McGurk 815 effect in a principled way, and provide a number of quantitative data about the dynamics 816 of this process. In the following, we will first discuss how these results fit inside the 817 binding and fusion architecture that we propose in the framework of audiovisual speech 
818 scene analysis. Then we will attempt to formalize this architecture in more detail, and

819 propose some elements of a cognitive model, to let emerge some open questions.

820 A. Characterization of the binding system in audiovisual speech 821 perception

\section{1. How context intervenes in audiovisual fusion}

823 The two experiments in this paper confirm the results of the two experiments presented in

824 our first study (Nahorna et al., 2012): the McGurk effect is not automatic, it depends on

825 the context provided by a sequence of audiovisual speech stimuli presented prior to the

826 McGurk target. Incoherent contexts of various types and durations decrease the amount

827 of fusion responses "da" in favor of auditory responses "ba", compared to coherent

828 contexts. This shows that there must exit in the audiovisual speech perception system a

829 device assessing audiovisual coherence and probably computing an audiovisual

830 coherence index of some kind: let us call this device a coherence box.

831 This coherence box is likely to be instrumental in the audiovisual speech detection

832 advantage (see Section I). Indeed, this advantage increases with the correlation between

833 visual cues (e.g. lip area or mouth opening) and audio cues (e.g. spectral features or

834 amplitude) (e.g. Grant and Seitz, 2000. Kim and Davis, 2004). It could also provide the

835 basis for audiovisual predictions, that is enable some predictions about the auditory

836 stream from the visual input, which has been proposed to be the basis for early

837 audiovisual interactions in evoked response potentials (e.g. van Wassenhove et al., 2005:

838 Arnal et al., 2009). We assume more generally that the computation of audiovisual

839 coherence index is a basic component in the audiovisual speech scene analysis system.

840 This index would enable the brain to evaluate the coherence between auditory and visual 
841 features in a complex multi-speaker scene, in order to properly associate the adequate

842 components inside a coherent audiovisual speech source. This is requested in a number

843 of experimental paradigms testing audiovisual speech perception in a scene associating

844 various faces and and/or various sounds (e.g. Andersen et al., 2009; Alsius and Soto-

845 Faraco, 2011).

846 It remains to understand how does this coherence box intervene in the decision process

847 leading to a given amount of fusion percepts in the present experiment. This is an open

848 question. Since this box supposedly enables the brain to know which auditory and visual

849 components must be associated to provide a fused percept (this is the binding problem),

850 our assumption is that a low coherence index provides low evidence for fusion and hence

851 decreases the visual weight in fusion, hence the increase in the amount of "ba" responses

852 for incoherent contexts in Experiment 1.

853 It could also be envisioned that context in these experiments intervenes as a post-

854 perceptual decision bias, according to which participants would be biased in their

855 decision to not report a fusion response when they receive evidence about an audiovisual

856 mismatch (provided by the context) ${ }^{(3)}$. However, the individual data show that the

857 decrease in fusions is not of an all or none type. For example, we observed that in

858 Experiment 1, most subjects display an increase in the amount of "ba" responses in the

859 strongly incoherent context whatever their score in the coherent context condition.

860 Therefore the decision bias would obey complex quantitative rules, not so different from

861 a decrease in visual weight in a decision fusion process. Anyway, the global conclusion at

862 this stage is that (1) a coherence index seems to be evaluated by the subject, and (2) its

863 value seems to modulate the subject's decision in some way. This is captured by the

864 formula proposed in Eq. (4) in the Introduction, and it is globally compatible with the

865 binding and fusion architecture: binding is realized by the coherence box through the 
866

867

868

869

870

871 incoherence of the audio and video streams leads the subjects to selectively decrease the

872 role of the visual input in the fusion process. The general hypothesis is that modulation is 873

874 auditory and visual input.

875 We begin to characterize the binding stage in the present paper. Firstly, Experiment 1 876 shows that the dynamics of unbinding is rapid. One syllable or the equivalent duration

877 (around $0.5 \mathrm{~s}$ ) suffices to produce a maximum decrease in the McGurk effect (around $87850 \%$ decrease). There even appears a trend, confirmed in Experiment 2, according to 879 which short durations of incoherence produce more unbinding than longer ones. The 880 interpretation of this fact is not completely clear. It could be due to a kind of adaptation 881 effect according to which the computation of coherence would include temporal 882 derivatives, enhancing the incoherence index at the beginning of an incoherent sequence.

883 Experiment 1 also confirms that pure phonetic incoherence suffices to produce an effect 884 on binding, since there is a difference between a coherent and a phonetically incoherent 885 context - with a significantly smaller McGurk effect in the second case. This means that 886 audiovisual correlations in time between audio and visual cues are probably not the 887 single elements that intervene in the assessment of audiovisual coherence, and that the 888 phonetic content of the incoming information also plays a part in this process. 
890 Experiment 2 shows that unbinding processes can be followed by rebinding processes, in

891 which coherent reset sets back the weight of the visual input and hence enables to recover 892 the McGurk effect. However, rebinding appears slower than unbinding, since it requires 893 at least 3 coherent syllables (for a duration around $1.5 \mathrm{~s}$ ) to be complete. The 894 interpretation seems to be that loosing faith in the common origin of the sound and face 895 seems rapid, but recovering faith implies to gather a minimum amount of new coherent 896 cues, which takes a longer time for accumulation of adequate information.

\section{3. Binding states and reset processes}

898 It is classically considered that auditory scene analysis involves a default grouped state 899 followed by a possible build-up of auditory segregation (Bregman, 1990). The systematic 900 bias towards the grouped interpretation is displayed both in the auditory and in the visual 901 modality (Hupé and Pressnitzer, 2012). In the case of multisensory scenes, a general 902 compatibility bias is displayed in various experiments dealing with the fusion of 903 conflicting cues (e.g. Yu et al., 2009; Noppeney et al., 2010). This bias suggests that 904 subjects suppose at the beginning of the task that the various cues are not conflicting 905 before evidence of conflict progressively leads the subjects to select one cue rather than 906 the other.

907 The present data are consistent with the hypothesis of a default state of the audiovisual 908 binding mechanism in which audio and video components are fused together. Various 909 evidence point towards this hypothesis. Firstly the existence of the McGurk effect itself 910 seems to require this assumption. Indeed, McGurk stimuli are just a specific case of 911 phonetic incoherence, not different from those used in Experiment 1. The fact that they 912 can be fused together implies that subjects process these stimuli under the underlying 
913 assumption of a default state. Notice that this underlying assumption is strong enough to

914 resist to a number of incongruence in the components of the sensory streams: 915 discrepancies in the spatial localisation of the auditory and visual sources (Bertelson et 916 al., 1994), temporal asynchronies (van Wassenhove et al., 2007), and even incoherence of 917 source identity, with a female face dubbed on a male voice (Green et al., 1991).

918 However, as we discussed at the end of Experiment 1 (Section II.C), our data do not 919 allow to know for sure whether binding is maximal with no context (and hence cannot be 920 increased by applying a coherent context, whatever its duration), or if it is actually sub921 optimal, in which case coherent context could increase the confidence that the auditory 922 and visual streams refer to a single source and hence the visual input would play a larger 923 role in the decision process. A challenge for future studies will be to better understand 924 how the evaluation of audiovisual coherence, and hence the amount of binding and the 925 weight of the visual input, are constantly updated along the flow of audiovisual 926 information.

927 A striking result of Experiment 2 is that a fixed reset has almost no rebinding effect, with 928 the consequence that even for the longest duration (around 1.5s) the subjects stay frozen 929 in an unbound state where the McGurk effect is largely decreased. It remains to study 930 how the subjects come back to their default bound state. The fact that the influence of 931 one stimulus on the next one seems rather weak (see Section II.B.3) makes us wonder 932 whether giving a response also resets the system. However, as discussed in that section, 933 there are too many confounding factors (associated to recalibration and contrast 934 mechanisms producing decision biases), which impede to answer to this question at this 935 stage.

936 A reset material should engage the subject into the understanding that the situation has 
937 dramatically changed. This could involve changing from one speaker to another, 938 assessing whether a piece of incoherent context from one speaker would modify the

939 McGurk effect for another speaker. Another question deals with the speech-specific

940 nature of the audiovisual binding system, asking whether for example an incoherent

941 audiovisual context made of non-speech material would be as efficient as the kind of

942 incoherent context used in the present study to reduce the McGurk effect.

\section{4. Response is global, response time seems local}

944 Reaction times to McGurk stimuli are seldom reported. When data are provided, they 945 display longer reaction times for incongruent (McGurk) stimuli compared to congruent 946 ones (e.g. Massaro and Cohen, 1983; Keane et al., 2010). Globally, there is a trend for 947 having longer reaction times for incongruent than for congruent audiovisual stimuli (see a 948 review in Tiippana et al., 2011). However, there are two possible interpretations of this 949 fact. Firstly, ambiguity in categorical judgment classically increases response latency in a 950 binary choice, and this is also in line with models of perceptual decision (e.g. Ratcliff and 951 Rouder, 1998; Smith and Ratcliff, 2004). Since incongruence generally results in more 952 ambiguous decisions, this should lead to longer response times. Secondly, it could also be 953 proposed that subjects are slower to respond to the extent that the auditory and visual 954 information give conflicting information about the speech event. These two assumptions 955 were discussed by Massaro and Cohen (1983), with the conclusion that perceptual 956 ambiguity was a better predictor of response times.

957 The results of the two experiments in this paper show that response times differ between 958 congruent "ba" and incongruent McGurk targets but do not depend on context. In

959 Experiment 1, response times are $58.3 \mathrm{~ms}$ larger for McGurk targets with no significant 960 effect of context type and duration, though responses vary between $50 \%$ "ba" for 
961 coherent context up to more than $80 \%$ "ba" for strongly incoherent context at the

962 smallest durations (1 or 2 syllables; see Figure 3). In Experiment 2, response times are $96349.5 \mathrm{~ms}$ larger for McGurk targets with no significant effect of reset type and duration, 964 though responses vary once again between $50 \%$ and 80 "ba" depending on the reset 965 condition (see Figure 8).

966 Therefore, the present data suggest that ambiguity is not the sole determinant of response 967 times for McGurk stimuli embedded in the various contextual environments that we used 968 here. Indeed, while responses are modulated by context and hence appear as the product 969 of a global computation where both context (including reset) and target play a role, 970 response times appear as mainly governed by the local characteristics of the target, with 971 quicker responses for congruent compared to incongruent targets.

973 B. Elements of a cognitive model

974 The various elements summarized in the previous section may be encapsulated within a 975 tentative cognitive architecture displayed in Figure 10. This architecture has no ambition 976 to be definitive or complete, it simply aims at making clear some basic components that 977 emerge from both the first study by Nahorna et al. (2012) and the present one. This 978 architecture comprises the following element, that we progressively define starting from 979 the standard model of Section I.

980 - Audiovisual fusion for decision. The links between auditory and visual inputs and 981 the decision box provide the basic architecture in all audiovisual fusion models 982 since thirty years. Restricting the architecture to this box provides the basis for Eq. 983 $(1)$. 
- Attentional processes and individual specificities. Fusion appears to depend on individual and cultural/linguistic factors and attentional processes. Adding the corresponding arrow towards the fusion box provides the basis for Eq. (3).

- Coherence $\mathrm{C}(\mathrm{t})$. Our experimental results on the role of context suggest that the brain constantly evaluates the coherence of the auditory and visual inputs to determine whether they belong to a coherent source. This participates in our view to a general audiovisual scene analysis process in which subjects determine in a complex scene which parts of the auditory information must be associated with which parts of the visual information. We recalled in Section I.B a number of natural candidates for the computation of coherence $\mathrm{C}(\mathrm{t})$ that could be based on computations of correlation or mutual information between such cues as global envelope or envelope in specific spectral bands for the audio input, and lip or face parameter cues for the visual input. The fact that phonetic incoherence suffices to modulate the McGurk effect suggests that phonetic cues also participate to the computation of local coherence $\mathrm{C}(\mathrm{t})$. The bidirectional arrows in Figure 10 between the auditory and visual boxes on one hand and coherence $\mathrm{C}(\mathrm{t})$ on the other hand indicate that $\mathrm{C}(\mathrm{t})$ may also provide some feedback enabling better extraction of monosensory cues, as displayed by data on the audio-visual speech detection advantage (Grant and Seitz, 2000; Schwartz et al., 2004).

- Binding state. Our results also suggest that coherence enables to constantly monitor the binding state in the subject's brain, and that the binding state would play a role in the fusion-decision process: the less bound the binding state, the smaller the weight of vision in the fusion process. There seems to exist a default state which is bound to a certain extent, but it remains to know if coherent context 
may drive towards a state which would be "more bound" than the default state. If we continue in the view that the binding state may vary on a quantitative scale between less and more bound, quantitative data in the present study suggest that the time constant towards less bound is more rapid than towards more bound. It would be around one syllable (less than $0.5 \mathrm{~s}$ ) in the first case, and around three syllables (more than 1s) in the second case. Interestingly, a previous work by our team on audiovisual speech source separation based on statistical modeling of audiovisual coherence showed that $400 \mathrm{~ms}$ suffice to adequately associate one audio stream and one video stream in a mixture of two faces and voices (Sodoyer et al., 2004). This confirms that there is enough information in less than $0.5 \mathrm{~s}$ to determine if a sound and a face may be bound together or not. Last but not least, results of Experiment 2 show that once the system is put in an unbound state by incoherent audiovisual material, it may stay frozen in this state for a while (at least $1.5 \mathrm{~s}$ ) unless new evidence for coherence is provided. Altogether, the coherence and binding state boxes and the way they enter the fusion box provide the basis for Eq. (4).

- Response time (RT). While it is classically considered that response times mainly depend on the decision process, with larger response times for more ambiguous stimuli, the present study suggests that local coherence also plays a role in response times. Local incongruence in McGurk targets would be detected by the subjects and slower their response. This is in line with various studies in which it appears that subjects are both able to perceive and estimate the discrepancy between the sight and the sound of a speaking face and fuse the two inputs into a single percept (Manuel et al., 1989; Summerfield and McGrath, 1984; SotoFaraco and Alsius, 2007, 2009). This suggests that the subjects have conscious 
1043 This set of experiments confirms that context may modify the McGurk effect, through 1044 a series of mechanisms, which combine unbinding (through incoherent context 1045 decreasing the role of the visual input) and rebinding (through coherent reset setting 1046 back the weight of the visual input). A first experiment displayed rapid unbinding 
1047 effects, with a reduction of the McGurk effect by half for very short incoherent 1048 contexts, made of one acoustic syllable dubbed on incoherent visual material extracted 1049 from the production of free sentences. A smaller incoherence amount, in which the 1050 phonetic content of the audio and video streams are different while keeping a perfect 1051 synchrony between the dynamics of sound and lips, resulted in a smaller but 1052 significant reduction of the McGurk effect compared with coherent context.

1053 A second experiment tested the role of possible reset stimuli after a period of 1054 incoherence producing strong unbinding. It showed that a fixed reset (acoustic silence 1055 plus fixed image of the speaker's face) has almost no rebinding effect, with the 1056 consequence that even for the longer duration (around 1.5s) the subjects stay frozen in 1057 an unbound state where the McGurk effect is largely decreased. On the contrary, a 1058 coherent reset of 3 syllables is enough to completely recover from unbinding and 1059 restore the default binding stage.

1060 Altogether these data can be captured inside a two-stage cognitive architecture in 1061 which a first binding stage assessing the coherence between sound and face would 1062 control the output of the fusion process and accordingly change the nature of the 1063 percept. Unbinding would result in a smaller role of vision in the decision process. 1064 Major challenges will involve a better understanding of possible binding states in the 1065 human's brain, in terms of online dynamics, neural correlates and changes in relation 1066 with age and hearing status. 
1069

1070 


\section{Acknowledgments}

1072 This work was supported by the French National Research Agency (ANR) through 1073 funding for the MULTISTAP project (MULTISTability and binding in Audition and 1074 sPeech: ANR-08-BLAN-0167 MULTISTAP). The research leading to these results has 1075 received funding from the European Research Council under the European Community's 1076 Seventh Framework Programme (FP7/2007-2013 Grant Agreement no. 339152). 
1078 Endnotes

1079

1080 (1) Corresponding author (jean-luc.schwartz@gipsa-lab.grenoble-inp.fr)

1081

1082 (2) Examples of stimuli for Experiments 1 and 2 are available at http://www.gipsa-

1083 lab.grenoble-inp.fr/ jean-luc.schwartz/fichiers_public_JLS/AV_Binding_demo/AV_Binding_Demo.html

1084

1085 (3) We thank one of the reviewers for having suggested this possible interpretation of our 1086 data.

1087

1088 
1089

1090

1091

1092

1093

1094

1095

1096

1097

1098

1099

1100

1101

1102

1103

1104

1105

1106

1107

\section{References}

Alsius, A., \& Munhall, K. (2013). "Detection of audiovisual speech correspondences without visual awareness," Psychological Science24, 423-31.

Alsius, A., Navarra, J., Campbell, R., \& Soto-Faraco, S.S. (2005). "Audiovisual integration of speech falters under high attention demands," Current Biology 15, 839-843.

Alsius, A., Navarra, J. \& Soto-Faraco, S. (2007). "Attention to touch weakens audiovisual speech integration," Experimental Brain Research 183, 399-404.

Alsius, A., \& Soto-Faraco S. (2011). "Searching for audiovisual correspondence in multiple speaker scenarios," Experimental Brain Research 213, 175-183.

Andersen, T.S., Tiippana, K., Lampinen, J. and Sams, M. (2001). "Modelling of Audiovisual Speech Perception in Noise," Proceedings of the Fourth International ESCA ETRW Conference on Auditory-Visual Speech Processing, Ålborg, Denmark, pp. 172-176.

Andersen, T.S., Tiippana, K., Laarni, J., Kojo I., \& Sams, M. (2009). "The role of visual spatial attention in audiovisual speech perception," Speech Communication 51,184193.

Arnal, L.H., Morillon, B., Kell, C.A. \& Giraud, A.-L. (2009). "Dual neural routing of visual facilitation in speech processing," Journal of Neuroscience 29, 13445-13453 
1108 Benoit, C., Mohamadi, T. \& Kandel, S., (1994). "Effects of phonetic context on audiovisual intelligibility of French," Journal of Speech and Hearing Research, 37, 11951203.

1111 Bernstein, L. E., Auer, E. T., \& Moore, J. K. (2004). “Audiovisual speech binding: convergence or association?," in G.A. Calvert, C. Spence C, \& B.E. Stein (eds.) The handbook of multisensory processes (pp 203-224). Cambridge: The MIT Press.

1114 Bernstein, L.E., Lu, Z.L., \& Jiang, J. (2008). "Quantified acoustic-optical speech signal 1115 incongruity identifies cortical sites of audiovisual speech processing," Brain Research

1117 Bertelson, P., Vroomen, J., De Gelder, B. (2003). "Visual recalibration of auditory speech identification: a McGurk aftereffect," Psychological Science 14, 592-597.

1119 Bertelson, P., Vroomen, J., Wiegeraad, G., \& de Gelder, B. (1994). "Exploring the relation between McGurk interference and ventriloquism," in Proc. ICSLP 94 (Vol.

1122 Berthommier, F. (2004). "A phonetically neutral model of the low-level audiovisual 1123 interaction," Speech Communication 44, 31-41.

1124 Besle, J., Fort, A., Delpuech, C., \& Giard, M.-H. (2004). "Bimodal speech: early 1125 suppressive visual effects in human auditory cortex," European Journal of $1126 \quad$ Neuroscience 20, 2225-2234.

1127 Bregman, A. S. (1990). Auditory scene analysis (773 p.), MIT Press: Cambridge, MA.

1128 Bregman, A.S. \& Pinker, S. (1978). "Auditory streaming and the building of timbre," 1129 Canadian Journal of Psychology 32, 19-31. 
1130 Cathiard, M.A., Schwartz, J.L., \& Abry, C. (2001). "Asking a naive question about the 1131 McGurk Effect: why does audio $[\mathrm{b}]$ give more [d] percepts with visual $[\mathrm{g}]$ than with visual [d]?," Proceedings AVSP-2001, 138-142.

1133 Colin, C., Radeau, M., Soquet, A., Demolin, D., Colin, F., \& Deltenre, P. (2002).

1134 "Mismatch negativity evoked by the McGurk-MacDonald effect: A phonetic 1135 representation within short-term memory," Clinical Neurophysiology 113, 495-506.

1136 Erber, N.P. (1969). "Interaction of audition and vision in the recognition of oral speech 1137 stimuli," Journal of Speech and Hearing Research 12, 423-425.

1138 Eskelund, K., Tuomainen, J., \& Andersen, T. S. (2011). Multistage audiovisual 1139 integration of speech: dissociating identification and detection," Experimental Brain $1140 \quad$ Research 208, 447-57.

1141 Fuster-Duran, A. (1995). "McGurk effect in Spanish and German listeners. Influences of 1142 visual cues in the perception of Spanish and German conflicting audio-visual 1143 stimuli," in Proceedings of the Eurospeech 95, pp. 295-298.

1144 Grant, K. W., \& Seitz, P. (2000). "The use of visible speech cues for improving auditory 1145 detection of spoken sentences," Journal of the Acoustical Society of America 108, $1146 \quad$ 1197-1208.

1147 Green, K., Kuhl, P., Meltzoff, A., \& Stevens, E. (1991). "Integrating speech information 1148 across talkers, gender, and sensory modality: female faces and male voices in the 1149 McGurk effect," Perception and Psychophysics 50, 524-536. 
1150 Heckmann, M., Kroschel, K., Savariaux, C., Berthommier, F. (2002). DCT-Based video 1151 features for audio-visual speech recognition. In: Proc. ICSLP02, Denver, pp. 1925$1152 \quad 1928$.

1153 Hupé, J.M., \& Pressnitzer, D. (2012). "The initial phase of auditory and visual scene analysis," Philosophical Transactions of the Royal Society B 367, 942-953.

1155 Huyse, A., Berthommier, F. et Leybaert, J. (2013). "Degradation of labial information modifies audiovisual speech perception in cochlear-implanted children," Ear and Hearing 34, 110-121.

Keetels, M., Stekelenburg, J., \& Vroomen, J. (2007). “Auditory grouping occurs prior to intersensory pairing: Evidence from temporal ventriloquism," Experimental Brain Research180, 449-456.

Keane, B. P., Rosenthal, O., Chun, N. H. \& Shams, L. (2010). “Audiovisual integration in high functioning adults with autism," Research in Autism Spectrum Disorders 4,

1164 Kim, J., Davis, C. (2003). "Hearing foreign voices: does knowing what is said affect masked visual speech detection," Perception 32, 111-120.

1166 Kim, J., \& Davis, C. (2004). "Investigating the audio-visual detection advantage," 1167 Speech Communication 44, 19-30.

1168 Lallouache, M.T. (1990). «Un poste 'visage-parole'. Acquisition et traitement de 1169 contours labiaux. (A "face-speech" workstation. Acquisition and processing of labial 1170 contours)," Proceedings XVIII Journées d'Etudes sur la Parole (pp. 282-286), 1171 Montréal. 
1172 van Maanen, L., Grasman, R.P.P.P., Forstmann, B.U., \& Wagenmakers, E-J. (2012), 1173 "Piéron's Law and optimal behavior in perceptual decision-making," Frontiers in 1174 Decision Neuroscience 5, 143.

1175 Manuel, S., Repp, B. H., Liberman, A. M., \& Studdert-Kennedy, M. (1989). "Exploring 1176 the "McGurk effect"," Paper presented at the 24th meeting of the Psychonomic Society, San 1177 Diego.

1178 Massaro, D. W. (1989). "Multiple Book Review of Speech Perception byEar and Eye: A 1179 Paradigm for Psychological Inquiry," Behavioral and Brain Sciences12, 741-794.

1180 Massaro, D. W. (1987). "Speech perception by ear and eye" (320 p.), Hillsdale: LEA.

1181 Massaro, D. W., \& Cohen, M. M. (1983). "Evaluation and Integration of Visual and 1182 Auditorial Information in Speech Perception," Journal of Experimental Psychology: 1183 Human Perception and Performance 9, 753-771.

1184 Massaro, D.W., Tsuzaki, M., Cohen, M.M., Gesi, A., Heredia, R. (1993). "Bimodal 1185 speech perception: an examination across languages," Journal of Phonetics 21, 445-

1187 McGurk, H., \& MacDonald, J. (1976). "Hearing lips and seeing voices," Nature 265, $1188 \quad 746-748$.

1189 Nahorna, O., Berthommier, F., \& Schwartz, J.L. (2012). "Binding and unbinding the 1190 auditory and visual streams in the McGurk effect," J. Acoust. Soc. Am. 132, 10611191 1077. 
1192 Noppeney, U., Ostwald, D., \& Werner, S. (2010). "Perceptual decisions formed by 1193 accumulation of audiovisual evidence in prefrontal cortex," Journal of Neuroscience 30, 7434-46.

1195 Ratcliff, R., \& Rouder, J.N. (1998). "Modeling response times for two-choice decisions," $1196 \quad$ Psychological Science 9, 347-356.

1197 Sanabria, D., Soto-Faraco, S., Chan, J.S., \& Spence, C. (2005). "Intramodal perceptual 1198 grouping modulates multisensory integration: Evidence from the crossmodal congruency task," Neuroscience Letters377, 59-64.

Schwartz, J. L. (2006). "Bayesian model selection: The $0 / 0$ problem in the fuzzy-logical model of perception," Journal of the Acoustical Society of America 120, 1795-1798.

Schwartz, J. L. (2010). "A reanalysis of McGurk data suggests that audiovisual fusion in 1203 speech perception is subject-dependent," Journal of the Acoustical Society of America 127, 1584-1594.

Schwartz, J.L., Tiippana, K., \& Andersen, T. (2010). "Disentangling unisensory from fusion effects in the attentional modulation of McGurk effects: a Bayesian modeling study suggests that fusion is attention-dependent," in Proceedings AVSP2010 (pp.

Schwartz, J.L., Berthommier, F., \& Savariaux, C. (2004). "Seeing to hear better: Evidence for early audio-visual interactions in speech identification," Cognition 93, B69-B78.

1212 Schwartz, J.L., Robert-Ribes, J., \& Escudier, P. (1998). “Ten years after Summerfield ... 1213 a taxonomy of models for audiovisual fusion in speech perception," in R. Campbell, 
B. Dodd \& D. Burnham (eds.) Hearing by Eye, II. Perspectives and directions in research on audiovisual aspects of language processing (pp. 85-108). Hove (UK): Psychology Press.

Sekiyama, K. \& Burnham, D. (2008). Impact of language on development of auditoryvisual speech perception. Developmental Science 11, 306-320.

Sekiyama, K. \& Tohkura, Y. (1993). "Inter-language differences in the influence of visual cues in speech perception," Journal of Phonetics 21, 427-444.

Sekiyama, K. \& Tohkura, Y. (1991). "McGurk effect in non-English listeners: Few visual effects for Japanese subjects hearing Japanese syllables of high auditory intelligibility," The Journal of the Acoustical Society of America 90, 1797-1805.

Smith, P.L., \& Ratcliff, R. (2004). "Psychology and neurobiology of simple decisions," Trends in Neurosciences 27, 161-168.

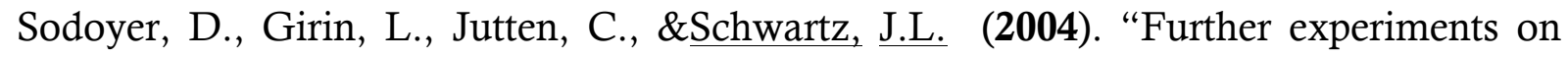
audio-visual speech source separation," Speech Communication44, 113-125.

Soto-Faraco, S., \& Alsius, A. (2007). "Conscious access to the unisensory components of a cross-modal illusion," Neuroreport 18, 347-50.

Soto-Faraco, S., \& Alsius, A. (2009). "Deconstructing the McGurk-MacDonald illusion," Journal of Experimental Psychology: Human perception and performance $35,580-7$.

Soto-Faraco, S., Navarra, J., \& Alsius, A. (2004). "Assessing automaticity in audiovisual speech integration: evidence from the speeded classification task," Cognition 92, B13-B23. 
1236 Sumby, W., \& Pollack, I. (1954). "Visual contribution to speech intelligibility in noise," Journal of the Acoustical Society of America26, 212-215.

1238

1239

1240

1241

1242

1243

1244

1245

1246

1247

1248

1249

1250

1251

1252

1253

1254

1255

1256

1257

Summerfield, Q. (1987). "Some preliminaries to a comprehensive account of audio-visual speech perception," inB. Dodd \& R. Campbell (eds.) Hearing by Eye: The Psychology of Lipreading (pp. 3-51) New York (NY): LawrenceErlbaum Associates.

Summerfield, Q., \& McGrath, M. (1984). "Detection and resolution of audio-visual incompatibility in the perception of vowel," Quarterly Journal of Experimental Psychology 36A, 51-74.

Tiippana, K., Andersen, T.S., \& Sams, M. (2004). "Visual attention modulates audiovisual speech perception," European Journal of Cognitive Psychology 16, 457472.

Tiippana, K. Puharinen, H., Möttönen, R., \& Sams, M. (2011). "Sound Location Can Influence Audiovisual Speech Perception When Spatial Attention Is Manipulated," Seeing and Perceiving 24, 67-90.

Vroomen, J. \& Baart, M. (2011). "Phonetic recalibration in audiovisual speech," in M. M. Murray \& M. T. Wallace (eds.) Frontiers in the neural basis of multisensory processes (pp. 363-379) Routledge: Taylor \& Francis.

van Wassenhove, V., Grant, K.W., \& Poeppel, D. (2005). "Visual speech speeds up the neural processing of auditory speech," Proceedings of the National Academy of Sciences 102, 1181-1186.

Van Wassenhove, V., Grant, K.W., \& Poeppel, D. (2007). "Temporal window of integration in bimodal speech," Neuropsychologia 45, 598-607. 
1258 Yu, A. J., Dayan, P., \& Cohen, J. D. (2009). Dynamics of attentional selection under 1259 conflict: Toward a rational Bayesian account. Journal of Experimental Psychology: 1260 Human Perception and Performance 35, 700-717.

1261

1262 


\section{Figure captions}

1264

1265 Figure 1 - Organization of stimuli in Experiment 1.

1267 Figure 2 - Percentage of "ba" responses (relative to the total number of "ba" + "da"

1268 responses) for the two targets in the three contexts and without context.

1269

1270 Figure 3 - Percentage of "ba" responses for McGurk targets for the three contexts and

1271 their five durations, compared to targets without context.

1273 Figure 4 - Effect of the preceding decision in Experiment 1. Responses to McGurk 1274 stimuli depending on context ("Coh" for coherent, "Incoh" for incoherent), preceding 1275 context ("Prec coh" for coherent preceding context, "Prec incoh" for incoherent 1276 preceding context), preceding target stimulus ("Prec ba" vs "Prec McGurk") and 1277 previous answer ("Ans ba" for previous "ba" target, "Ans ba" and "Ans da" for previous 1278 "McGurk" target). We do not present results for phonetically incoherent context to make 1279 the figure clearer.

1281 Figure 5- Mean response times for the two targets in the three contexts and without 1282 context. 
1284 Figure 6 - Mean response times for the two targets in the five context durations and 1285 without context.

1287 Figure 7 - Organization of stimuli in Experiment 2.

1289 Figure 8 - Percentage of "ba" responses (relative to the total number of "ba" + "da" 1290 responses) for the McGurk targets with coherent context and with incoherent context for 1291 the two reset types and the four reset durations.

1293 Figure 9 - Mean response times for the two targets in the two reset conditions.

1295 Figure 10 - A possible cognitive architecture for audiovisual binding and fusion in speech 1296 perception. 